\title{
Development of a Novel Model of Central Retinal Vascular Occlusion and the Therapeutic Potential of the Adrenomedullin-Receptor Activity-Modifying Protein 2 System
}

\author{
Kazutaka Hirabayashi, ${ }^{* \dagger}$ Masaaki Tanaka, ${ }^{* \dagger}$ Akira Imai, ${ }^{* \dagger}$ Yuichi Toriyama, ${ }^{* \dagger}$ Yasuhiro Iesato, ${ }^{* \dagger}$ Takayuki Sakurai, ${ }^{*}$ \\ Akiko Kamiyoshi, ${ }^{*}$ Yuka Ichikawa-Shindo, ${ }^{*}$ Hisaka Kawate, ${ }^{*}$ Megumu Tanaka, ${ }^{*}$ Kun Dai, ${ }^{*}$ Nanqi Cui, ${ }^{*}$ Yangxuan Wei, ${ }^{*}$ \\ Keisei Nakamura, ${ }^{*}$ Shiho Iida, ${ }^{*}$ Shuhei Matsui, ${ }^{*}$ Akihiro Yamauchi, ${ }^{\S}$ Toshinori Murata, ${ }^{\dagger}$ and Takayuki Shindo*
}

From the Department of Cardiovascular Research,* Shinshu University Graduate School of Medicine, Nagano; the Departments of Ophthalmology ${ }^{\dagger}$ and Anesthesiology, ${ }^{\ddagger}$ Shinshu University School of Medicine, Nagano; and the Research Center, ${ }^{\S}$ Japan Bio Products Co, Ltd, Tokyo, Japan

\author{
Accepted for publication \\ October 23, 2018. \\ Address correspondence to \\ Takayuki Shindo, M.D., Ph.D., \\ Department of Cardiovascular \\ Research, Shinshu University \\ Graduate School of Medicine, \\ Asahi 3-1-1, Matsumoto, \\ Nagano 390-8621, Japan. E- \\ mail: tshindo@shinshu-u.ac.jp.
}

\begin{abstract}
Central retinal vein occlusion (CRVO) is an intractable disease that causes visual acuity loss with retinal ischemia, hemorrhage, and edema. In this study, we developed an experimental CRVO model in mice and evaluated the therapeutic potential of the pleiotropic peptide adrenomedullin (ADM) and its receptor activity-modifying protein 2 (RAMP2). The CRVO model, which had phenotypes resembling those seen in the clinic, was produced by combining i.p. injection of Rose bengal, a photoactivator dye enhancing thrombus formation, with laser photocoagulation. Retinal vascular area, analyzed using fluorescein angiography and fluorescein isothiocyanate-perfused retinal flat mounts, was decreased after induction of CRVO but gradually recovered from day 1 to 7 . Measurements of retinal thickness using optical coherence tomography and histology revealed prominent edema early after CRV0, followed by gradual atrophy. Reperfusion after CRV0 was diminished in Adm and Ramp2 knockout (KO) mice but was increased by exogenous ADM administration. CRVO also increased expression of a coagulation factor, oxidative stress markers, and a leukocyte adhesion molecule in both wild-type and $A d m \mathrm{KO}$ mice, and the effect was more pronounced in Adm KO mice. Using retinal capillary endothelial cells, ADM was found to directly suppress retinal endothelial injury. The retinoprotective effects of the Adm-Ramp2 system make it a novel therapeutic target for the treatment of CRV0. (Am J Pathol 2019, 189: 449-466; https://doi.org/10.1016/j.ajpath.2018.10.021)
\end{abstract}

Retinal vein occlusion (RVO) is the second most common retinal vascular disease, the first being diabetic retinopathy (DR). ${ }^{1}$ Obstruction of the retinal vein is commonly caused by thrombus formation, which can occur as a complication associated with hypertension, diabetic mellitus, and dyslipidemia, among others. RVO can be subdivided into central retinal vein occlusion $(\mathrm{CRVO})$ and branch retinal vein occlusion based on the site of the occlusion. In both the cases, retinal capillary dropout, ischemia, macular edema, and vascular complications initiated by the occlusion can lead to severe visual loss. But despite its relatively high prevalence, substantial controversy still surrounds the pathophysiology of RVO. ${ }^{2}$
The fundamental molecular pathogenesis of RVO is still under discussion, although vascular endothelial growth factor (VEGF) is thought to be a key player. This is because VEGF promotes hyperpermeability and inflammation of the

Supported by a Ministry of Education, Culture, Sports, Science and Technology, Japan KAKENHI grant-in-aid for scientific research, Core Research for Evolutional Science and Technology of Japan Science and Technology Agency, Japan Agency for Medical Research and Development (AMED), a National Cardiovascular Center grant for cardiovascular diseases (T.S.), a Takeda Medical Research Foundation grant (T.S.), a SENSHIN Medical Research Foundation grant (T.S.), a Naito Foundation grant (T.S.), a NOVARTIS Foundation (Japan) for the Promotion of Science grant (T.S.), and a Bristol-Myers Squibb research grant (T.S.).

Disclosures: A.Y. is an employee of Japan Bio Products Co., Ltd. 
retinal vasculature, as well as neovascularization. In recent years, intravitreal administration of anti-VEGF agents has been used to treat $\mathrm{RVO}^{3}$; however, this approach has several associated drawbacks. One is recurrence of macular edema; another is tachyphylaxis, which can occur after long-term anti-VEGF administration. ${ }^{4,5}$ And finally, anti-VEGF agents may not fully reverse the functional and structural damage caused by RVO. For those reasons, identification of other therapeutic targets involved in controlling vascular integrity would be desirable.

Originally isolated from human pheochromocytoma, adrenomedullin (ADM) is a vasodilating polypeptide whose expression is up-regulated under ischemic conditions. ${ }^{6-8}$ Homozygous $\mathrm{Adm}$ knockout $\left(\mathrm{Adm}^{--}\right)$mice die in utero because of vascular structural abnormalities, ${ }^{9}$ which is indicative of ADM's indispensability for proper vascular development. Moreover, ADM also exerts vascular effects in adults, ${ }^{9,10}$ and it is now known to be widely distributed in numerous tissues and organs and to exert a variety of physiological effects in addition to vasodilatation. For example, ADM also exerts antioxidant, anti-inflammatory, antifibrotic, and antiapoptotic effects. ${ }^{11-13}$ The main body of the ADM receptor is calcitonin receptor-like receptor (CLR), a seven-transmembrane domain G-protein-coupled receptor. CLR associates with one of the three subtypes of receptor activity-modifying protein (RAMP), which determines the affinity of CLR for its ligands. ${ }^{14,15}$ Homozygous Ramp2 knockout (Ramp $2^{-l-}$ ) mice die in utero and exhibit a phenotype similar to that of $A d m^{--}$mice, which suggests that the ADM-RAMP2 system is specifically involved in vascular development. ${ }^{16}$

$A d m$ expression has been also detected in the eye. In earlier reports, ADM was shown to act as a vasodilator in the retinal arteries and to increase choroidal blood flow and ophthalmic arterial flow velocity. ${ }^{17,18}$ In addition, ADM levels are elevated in the vitreous fluid of DR patients, ${ }^{19-21}$ and plasma and vitreous ADM levels are reportedly related to the severity and stability of DR. ${ }^{21,22}$ Collectively, these findings suggest that ADM is involved in the pathophysiology of ocular diseases. Also, the ADM-RAMP2 system was shown to be crucially involved in retinal angiogenesis using an oxygen-induced retinopathy model with heterozygous Adm and Ramp2 knockout (KO) mice. ${ }^{23,24}$ Exogenous administration of ADM suppresses vascular hyperpermeability and inflammation in a DR model. ${ }^{25}$ These observations prompted us to investigate the relationship between ADM and CRVO.

An animal model could help understand the precise pathophysiology of CRVO, which may serve as the basis for development of new treatments. However, there is currently no standard animal model in which the phenotypic characteristics exactly mimic human CRVO. This mainly reflects the complexity of the pathologic changes occurring along the time course of CRVO. Nevertheless, several animal models of vascular occlusion using laser photocoagulation, photodynamic coagulation, intravitreal injection of dermal fibroblasts, and diathermic cauterization have been proposed. ${ }^{26}$ Among them, the photodynamic coagulation method $^{27}$ shows early features of the clinical disease, including retinal capillary dropout, hemorrhage, and edema. Herein, we developed an easy and reproducible model of CRVO in mice, which is particularly useful when analyzing genetically modified mice. Using this model, the sequential events occurring in CRVO, including capillary dropout, ischemia, macular edema, and inflammation, were analyzed and the potential of ADM to serve as a therapeutic agent for the treatment of CRVO was assessed.

\section{Materials and Methods}

\section{Animals}

$A d m$ and Ramp2 KO mice were previously generated in our group. ${ }^{9,16}$ But because homozygous Adm or Ramp2 $\mathrm{KO}$ is embryonically lethal, heterozygous $A d m$ and Ramp2 KO mice were used. In these mice, expression of the affected genes is reduced to approximately half that in wild-type (WT) mice. The following mice were used in this study: 9- to 12week-old male Adm KO, Ramp2 KO, and WT mice, as well as WT mice systemically administered ADM using an osmotic pump. The background of all the mice was C57BL/6J. Before the operative procedures, the mice were anesthetized through i.p. injection of a combination anesthetic that included $0.3 \mathrm{mg} / \mathrm{kg}$ of medetomidine (Nippon Zenyaku Kogyo Co Ltd, Koriyama, Japan), $4.0 \mathrm{mg} / \mathrm{kg}$ of midazolam (Astellas Pharma Inc., Tokyo, Japan), and $5.0 \mathrm{mg} / \mathrm{kg}$ of butorphanol (Meiji Seika Pharma Co Ltd, Tokyo, Japan).

All animal handling procedures were in accordance with a protocol approved by the Ethics Committee of Shinshu University School of Medicine. All experiments were performed in accordance with the Association for the Research in Vision and Ophthalmology's Statement for the Use of Animals in Ophthalmic and Vision Research and our institutional guidelines.

\section{Administration of ADM to Mice}

Human ADM (Peptide Institute, Inc., Osaka, Japan) dissolved in phosphate-buffered saline (PBS) was infused into s.c. tissues using osmotic pumps (Alzet; DURECT Co, Cupertino, CA). The delivery rate was $29 \mu \mathrm{g} / \mathrm{kg}$ per day, and the mice received ADM for 7 days. Mice treated with PBS served as controls.

\section{Intravitreal Administration of ADM to Mice}

For subsequent study of CD68- and CD206-immunostained retinal flat-mount preparations, human ADM dissolved in PBS $\left(10^{-5} \mathrm{~mol} / \mathrm{L} ; 1.0 \mu \mathrm{L}\right)$ was intravitreally injected at the temporal corneoscleral junction using a Hamilton syringe fitted with a 32-gauge needle under a stereoscopic surgical microscope. As a control, eyes were intravitreally injected 
Table 1 Primers Used for Real-Time PCR

\begin{tabular}{|c|c|}
\hline Primer name/type & Primer sequence \\
\hline $\begin{array}{l}\text { m Calcrl (CLR) } \\
\text { forward }\end{array}$ & 5'-AGGCGTTTACCTGCACACACT-3' \\
\hline $\begin{array}{l}\text { m Calcrl (CLR) } \\
\text { reverse }\end{array}$ & 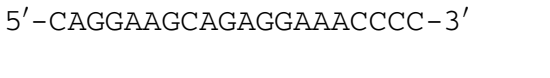 \\
\hline m Calcrl (CLR) probe & 5'-ATCGTGGTGGCTGTGTTTGCGGAG-3' \\
\hline m Ramp1 forward & 5'-GCACTGGTGGTCTGGAGGA-3' \\
\hline m Ramp1 reverse & 5'-СССТСАTCACCTGGGATACCT-3' \\
\hline m Ramp1 probe & $5^{\prime}$-CAAGCGCACAGAGGGCATCGTG-3' \\
\hline m Ramp2 forward & 5'-GCAGCCCACCTTCTCTGATC-3' \\
\hline m Ramp2 reverse & 5'-AACGGGATGAGGCAGATGG-3' \\
\hline m Ramp2 probe & $5^{\prime}$-CCCAGAGGATGTGCTCCTGGCCAT-3' \\
\hline m Ramp3 forward & $5^{\prime}-$ TGCAACGAGACAGGGATGC- $3^{\prime}$ \\
\hline m Ramp3 reverse & $5^{\prime}$-GCATCATGTCAGCGAAGGC-3' \\
\hline m Ramp3 probe & $5^{\prime}$-AGAGGCTGCCTCGCTGTGGGAA-3' \\
\hline $\begin{array}{l}\text { TaqMan m Adm } \\
\text { forward }\end{array}$ & $5^{\prime}$-CTACCGCCAGAGCATGAACC-3' \\
\hline $\begin{array}{l}\text { TaqMan m Adm } \\
\text { reverse }\end{array}$ & 5'-GAAATGTGCAGGTCCCGAA-3' \\
\hline $\begin{array}{l}\text { TaqMan m Adm } \\
\text { probe }\end{array}$ & 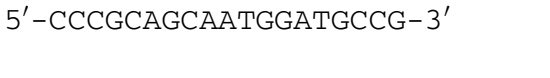 \\
\hline $\begin{array}{l}\text { SYBR m Adm } \\
\text { forward }\end{array}$ & 5'-GGACACTGCAGGGCCAGAT- $3^{\prime}$ \\
\hline SYBR $\mathrm{m} A d m$ reverse & 5'-GTAGTTCCCTCTTCCCACGACTTA-3' \\
\hline $\begin{array}{l}\text { m Serpine1 (PAI-1) } \\
\text { forward }\end{array}$ & 5'-ACCAAGAGCAGCTCTCTGTAGCA-3' \\
\hline $\begin{array}{l}\text { m Serpine1 (PAI-1) } \\
\text { reverse }\end{array}$ & 5'-CTTGGCCCATGAAGAGGATTGTCT-3' \\
\hline m Vcam-1 forward & 5'-CCCTGAATACAAAACGATCGC-3' \\
\hline m Vcam-1 reverse & 5'-CAGCCCGTAGTGCTGCAAG-3' \\
\hline m Cd68 forward & 5'-TGGCGGTGGAATACAATGTG-3' \\
\hline $\mathrm{m}$ Cd68 reverse & 5'-GAGATGAATTCTGCGCCATGA-3' \\
\hline $\begin{array}{l}\text { m Ncf1 (p47phox) } \\
\text { forward }\end{array}$ & 5'-АTCCTATCTGGAGCCCCTTGA-3' \\
\hline $\begin{array}{l}\text { m Ncf1 (p47phox) } \\
\text { reverse }\end{array}$ & $5^{\prime}$-CACCTGCGTAGTTGGGATCC-3' \\
\hline $\begin{array}{l}\text { m Ncf2 (p67phox) } \\
\text { forward }\end{array}$ & 5'-CAGACCCAAAACCCCAGAAA-3' \\
\hline $\begin{array}{l}\text { m Ncf2 (p67phox) } \\
\text { reverse }\end{array}$ & 5'-AAAGCCAAACAATACGCGGT-3' \\
\hline $\begin{array}{l}\text { m Nos3 (eNOS) } \\
\text { forward }\end{array}$ & $5^{\prime}-\mathrm{AGGCACTGCTGAGCCGAGT-3'}$ \\
\hline $\begin{array}{l}\mathrm{m} \text { Nos3 (eNOS) } \\
\text { reverse }\end{array}$ & $5^{\prime}$-TTCTCCAGTTGTTCCACAGCC-3' \\
\hline $\begin{array}{l}\text { m Tek (Tie-2) } \\
\text { forward }\end{array}$ & 5'-GGAACCTGACCTCGGTGCTA-3' \\
\hline $\begin{array}{l}\text { m Tek }(\text { Tie-2) } \\
\text { reverse }\end{array}$ & 5'-CTGCGCCTTGGTGTTGACT-3' \\
\hline m Angpt1 forward & $5^{\prime}$-CATGGGCAATGTGCCTACAC-3' \\
\hline m Angpt1 reverse & 5'-TCGCACTCTCACGGCAGTT-3' \\
\hline m $\operatorname{Tnf}-\alpha$ forward & $5^{\prime}$-ACGGCATGGATCTCAAAGAC- $3^{\prime}$ \\
\hline m Tnf- $\alpha$ reverse & $5^{\prime}$-AGATAGCAAATCGGCTGACG-3' \\
\hline $\begin{array}{l}\text { m Kdr (VEGFR2) } \\
\text { forward }\end{array}$ & 5'-ACTGCAGTGATTGCCATGTTCT-3' \\
\hline $\begin{array}{l}\text { m } K d r(\text { VEGFR2) } \\
\text { reverse }\end{array}$ & 5'-TCATTGGCCCGCTTAAGG-3' \\
\hline r Gapdh forward & 5'-AGACAGCCGCATCTTCTTGT-3' \\
\hline r Gapdh reverse & 5'-CTTGCCGTGGGTAGAGTCAT-3' \\
\hline
\end{tabular}

(table continues)
Table 1 (continued)

\begin{tabular}{|c|c|}
\hline Primer name/type & Primer sequence \\
\hline r Vcam-1 forward & 5'-GGGATTAATGAGGCTGGAAT-3' \\
\hline r Vcam -1 reverse & 5'-TGTCTCCTGTCTTGGCTTTC-3' \\
\hline $\begin{array}{l}\text { r Ccl2 (MCP-1) } \\
\text { forward }\end{array}$ & 5'-GAAAATCACAAGCAGCCAGT-3' \\
\hline $\begin{array}{l}\text { r Ccl2 }(\mathrm{MCP}-1) \\
\text { reverse }\end{array}$ & $5^{\prime}$-GGGGAAGGTCAGAGGAAATA-3' \\
\hline $\begin{array}{l}\text { r Ncf1 (p47phox) } \\
\text { forward }\end{array}$ & 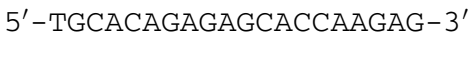 \\
\hline $\begin{array}{l}\text { r Ncf1 (p47phox) } \\
\text { reverse }\end{array}$ & 5'-GGTAGGAGCCTCTGACCTGA-3' \\
\hline $\begin{array}{l}\text { r Ncf2 (p67phox) } \\
\text { forward }\end{array}$ & $5^{\prime}$-CCCCACTCGAGGATTTGCTT-3' \\
\hline $\begin{array}{l}\text { r Ncf2 (p67phox) } \\
\text { reverse }\end{array}$ & $5^{\prime}-$ TCGCTGGAAGTAAGCCACTG-3' \\
\hline $\begin{array}{l}\text { Kdr (VEGFR2) } \\
\text { forward }\end{array}$ & $5^{\prime}$-GGGTTTTGTCAAGTGGCGAC-3' \\
\hline $\begin{array}{l}\text { r Kdr (VEGFR2) } \\
\text { reverse }\end{array}$ & $5^{\prime}$-ATCACGTGGCCACAAAGCTA-3' \\
\hline r Cd68 forward & 5'-ACAGTTTCTCCCACCACAAA-3' \\
\hline r Cd68 reverse & 5'-CCTGGGTCAGGTACAAGATG-3' \\
\hline
\end{tabular}

CLR, calcitonin receptor-like receptor; eNOS, endothelial nitric oxide synthase; m, mouse; MCP, monocyte chemotactic protein; PAI, plasminogen activator inhibitor; $r$, rat; Tie, tyrosine kinase with IgG and EGF homology domain; VEGFR, vascular endothelial growth factor receptor.

with $1.0 \mu \mathrm{L}$ of PBS. After the procedure, the mice were administered moxifloxacin hydrochloride antibiotic drops (Vegamox Ophthalmic Solution; Alcon, Fudenberg, Switzerland).

\section{Photodynamic Coagulation Method}

CRVO was induced in the right eye of each mouse. After anesthesia, $40 \mathrm{mg} / \mathrm{kg}$ Rose bengal (FUJIFILM Wako Pure Chemical Corp., Osaka, Japan) was intraperitoneally injected. The pupil was then dilated with one drop of $0.5 \%$ tropicamide and $0.5 \%$ phenylephrine (Mydrine P; Santen, Osaka, Japan). Photodynamic coagulation was induced using a green laser slit-lamp delivery system (GYC-1000; NIDEK, Gamagori, Japan) with a cover slip serving as a contact lens. The wavelength was $532 \mathrm{~nm}$, the power was 50 $\mathrm{mW}$, the duration was 3 seconds, and the spot size was 50 $\mu \mathrm{m}$ and positioned at the optic disc. Photocoagulation was repeated several times until central retinal vein occlusion was confirmed. After the CRVO procedure, $3.0 \mathrm{mg} / \mathrm{kg}$ atipamezole (ZEOAQ, Fukushima, Japan) was intraperitoneally injected.

\section{Fluorescence Angiography}

On days 1, 3, and 7 after induction of CRVO, mice were anesthetized, and the cornea was kept moist using saline. Mice were then manually held in front of a fundus camera (TRC-50AX; Topcon, Tokyo, Japan), and fluorescence angiography (FA) was performed after i.p. injection of 
$12 \mu \mathrm{L} / \mathrm{g} 2.5 \%$ fluorescein sodium (Alcon, Freiburg, Germany). Images were taken after 1 to 3 minutes of fluorescein perfusion and used for quantitative evaluations. Vascular density, vascular area, and vascular branch points were quantified from the FA images using AngioTool image analysis software version 0.6a (National Cancer Institute, Bethesda, MD).

\section{FITC-Dextran Perfusion and Retinal Flat Mount}

On day 7 after induction of CRVO, mice were anesthetized and perfused with $1 \mathrm{~mL}$ of PBS containing $50 \mathrm{mg} / \mathrm{mL}$ fluorescein isothiocyanate (FITC)-labeled dextran (molecular weight, $2 \times 10^{6}$; Sigma-Aldrich, St. Louis, MO) via the left ventricle. The eyes were then enucleated and fixed for 1 hour in $4 \%$ paraformaldehyde, after which the cornea and lens were removed, and the entire retina was carefully dissected from the eyecup. Four radial cuts were then made from the edge to the equator, and the eyecup (retina) was flat mounted with the scleral side facing down and examined using a fluorescence microscope. Images of whole mount retinas were taken using a fluorescence microscope (BZ-9000; Keyence, Osaka, Japan). Vascular area was quantified from the FITC images using AngioTool image analysis software.

\section{Topical Endoscopy Fundus Imaging}

Topical endoscopy fundus imaging was performed, as described previously, ${ }^{28}$ with some modifications. An endoscope coupled to a 5-cm-long otoscope with a 3-mm outer diameter (1218AA; Karl Storz, Tuttlingen, Germany) was used. A reflex digital camera with an 18-megapixel CMOS image sensor (EOS REBEL T4i; Canon, Tokyo, Japan) was connected to the endoscope through an adapter. Pupils were dilated using topical $0.5 \%$ phenylephrine and $0.5 \%$ tropicamide (Mydrin-P; Santen), which were respectively applied 60 and 30 minutes before eye examination. Mice were anesthetized just before the examination, after which their whiskers were shaved, and one drop of $0.4 \%$ oxybuprocaine (Nitto Medic, Tokyo, Japan) was applied to each eye. The camera was placed on a platform, and the endoscope was slowly moved toward the mouse. Once the endoscope was in contact with the gel covering the cornea, the photographer adjusted its position by horizontally displacing the tip. Focus and illumination were adjusted during examination of the fundus through the camera.

\section{Measurement of Retinal Edema Using OCT}

Optical coherence tomography (OCT) imaging was performed to evaluate retinal edema after induction of CRVO. Envisu R-Class OCT (Leica Microsystems, Wetzlar, Germany) and Heidelberg SPECTRALIS OCT (Heidelberg Engineering GmbH, Heidelberg, Germany) were used. With Envisu R-Class OCT, retina thickness at $0.25 \mathrm{~mm}$ from the
A Day 7
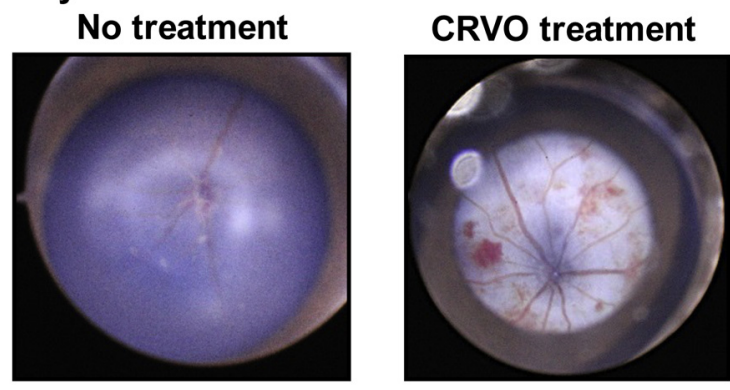

B Day 7

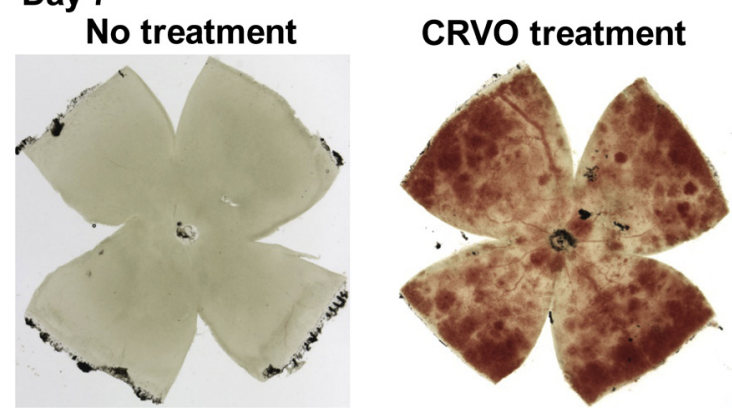

Figure 1 Evaluation of retinal hemorrhage in the central retinal vein occlusion (CRV0) model. A: Topical endoscopy fundus imaging showing the ocular fundus of untreated and CRV0-treated mice (day 7). In the CRVOtreated mice, note the retinal hemorrhage and venous dilatation with tortuousness resembling clinical CRV0. B: Retinal flat-mount images of untreated and CRV0-treated mice (day 7). Retinal hemorrhage is prominent in the CRVO-treated mice.

optic disc was measured before and after CRVO (days 1 to 20). With Heidelberg SPECTRALIS OCT, the Heidelberg Eye Explorer (Heidelberg Engineering $\mathrm{GmbH}$ ) segmentation algorithm was applied, which recognizes the internal limiting membrane and the basal membrane. Segmentation was confirmed by visual observation, and manual adjustments were made when the internal limiting membrane and basement membrane clearly deviated. For analysis of retinal thickness, a standard Early Treatment Diabetic Retinopathy Study (ETDRS) grid with circle diameters of 1, 3, and $6 \mathrm{~mm}$ was centered on the optic nerve head. Retinal volume within a region $6 \mathrm{~mm}$ in diameter was automatically calculated. Retinal volume was measured before and after CRVO (days 1 to $7,11,14,18$, and 21). OCT angiography was also performed using a SPECTRALIS OCT angiography module (Heidelberg Engineering $\mathrm{GmbH}$ ) before and after CRVO (days 7 and 21).

\section{Isolectin B4 Staining}

Retinas were isolated and stained overnight at $4{ }^{\circ} \mathrm{C}$ with Alexa Fluor 568-conjugated Griffonia bandeiraea simplicifolia isolectin B4 (Thermo Fisher Scientific, Waltham, MA) in PBS with 0.3\% Triton X-100 (1:200 dilution). After washing three times in PBS, the retinas were whole mounted with the photoreceptor side down on microscope 

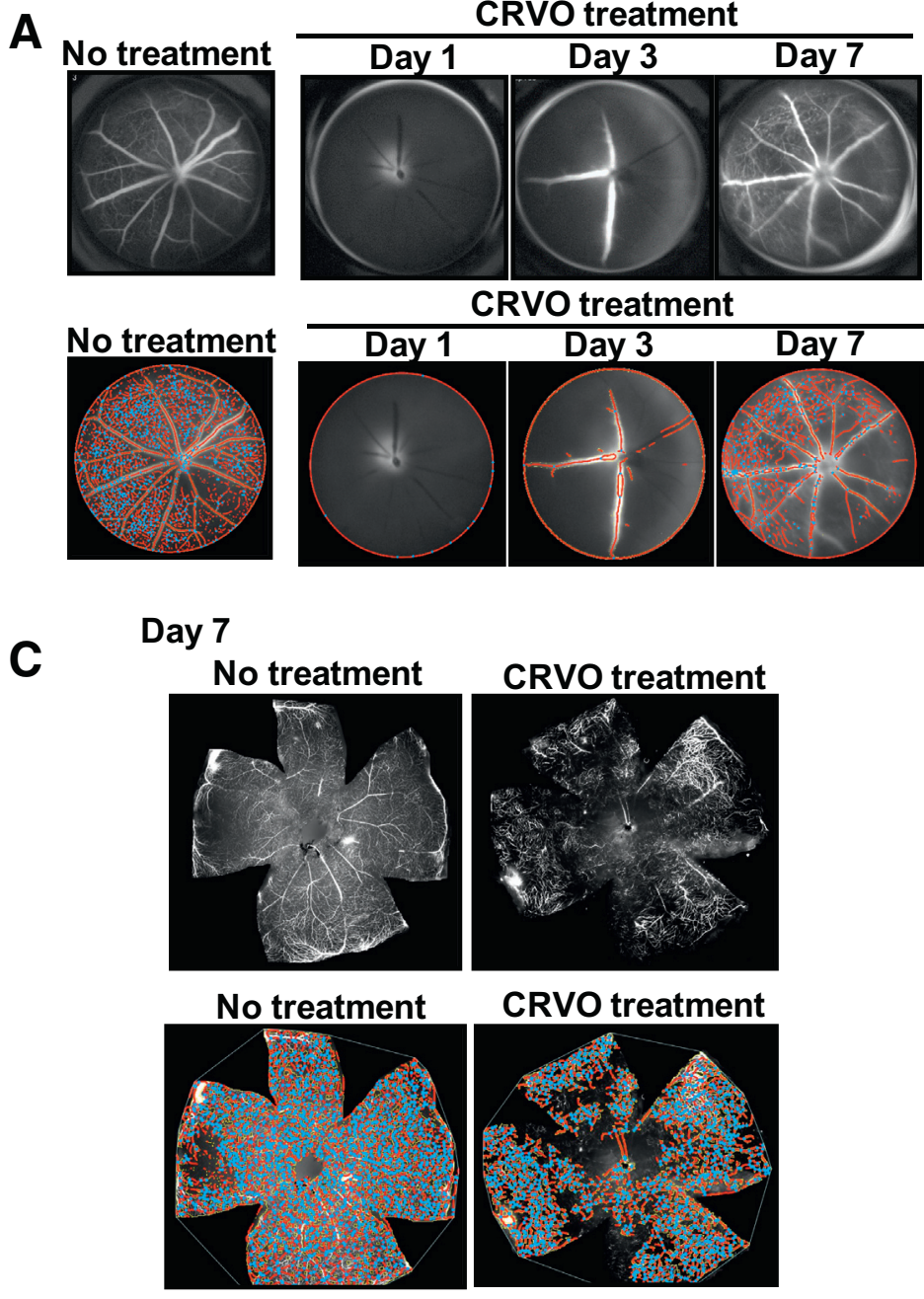

Figure 2 Evaluation of vascular perfusion in the central retinal vein occlusion (CRVO) model. A: Top row: Representative fluorescein angiography (FA) images from untreated control and CRV0-treated mice (days 1, 3, and 7). Bottom row: FA images were analyzed using AngioTool software version 0.6a . Blood vessels are depicted in red, and vascular branch points are depicted in blue. B: Percentage vascular areas in FA images quantified using AngioTool. A Dunnett's test was used. C: Top row: Fluorescein isothiocyanate (FITC)-perfused retinal flat-mount images from untreated control mice and CRV0-treated mice (day 7). Bottom row: FITC-perfused retinal flat-mount images were analyzed using AngioTool. Blood vessels are depicted in red, and vascular branches are depicted in blue. D: Percentage vascular area in FITC-perfused retinal flat-mount images on day 7 quantified using AngioTool. An unpaired $t$-test was used. Data are expressed as means \pm SEM. $n=6$ (B and D, CRVO); $n=5$ (D, untreated). ${ }^{*} P<0.05,{ }^{*} P<0.01$, and ${ }^{* * * *} P<0.0001$.

slides and embedded in fluorescence mounting medium (Agilent Technologies, Santa Clara, CA). Images of whole-mount retinas were taken using a fluorescence microscope (BZ-9000).

\section{Immunohistochemistry}

Mice were sacrificed, and retinal flat mounts were made as described above. After blocking with $1 \%$ bovine serum albu$\mathrm{min}$, the flat mounts were stained using rat anti-mouse F4/80 antibody (Bio-Rad, Hercules, CA), rat anti-mouse CD68 antibody (Abcam, Cambridge, UK), or rabbit anti-mouse CD206 antibody (Abcam), which were then reacted with appropriate secondary antibodies. CD68- or CD206-positive cells were then counted in multiple microscope fields.

Retinas were also fixed overnight in $4 \%$ paraformaldehyde, embedded in paraffin, and cut into sections
(5 $\mu \mathrm{m}$ thick) for hematoxylin and eosin staining and immunohistological analysis of rabbit anti-mouse p67phox antibody (Merck Millipore, Darmstadt, Germany). Images of whole-mount retinas and sections were taken using a fluorescence microscope (BZ-9000).

\section{Real-Time RT-PCR Array Analysis}

A PCR array ( $\mathrm{RT}^{2}$ Profiler PCR Array; Qiagen, Hilden, Germany) was used to assess expression of a focused panel of genes. After $1 \mu \mathrm{g}$ of total RNA of retina was converted to cDNA using a $\mathrm{RT}^{2}$ First Strand Kit (Qiagen), levels of specific transcripts were assessed using a Mouse Angiogenesis and Mouse Endothelial Cell Biology PCR Array (Qiagen), according to the manufacturer's protocols. All PCRs were run using a StepOnePlus Real-Time PCR System (Thermo Fisher Scientific). RT ${ }^{2}$ Profiler PCR Array 


\section{A \\ OCT color mapping}

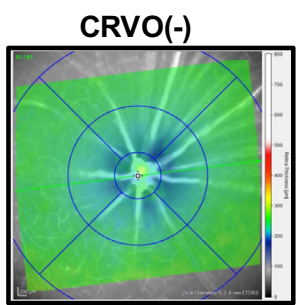

Day 1

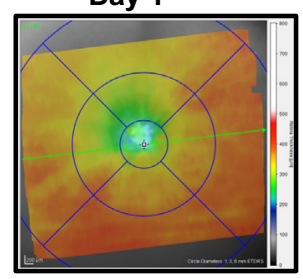

Day 3

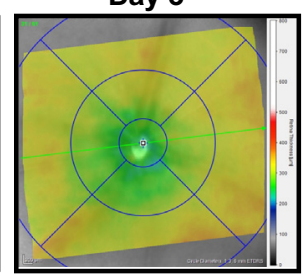

Day 7

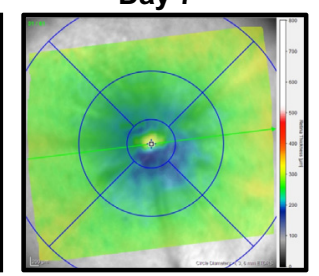

Day 21

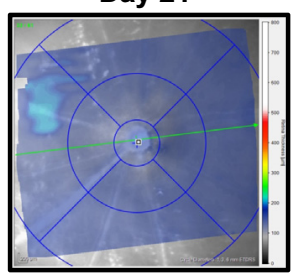

B

Cross section of retina (B-scan)

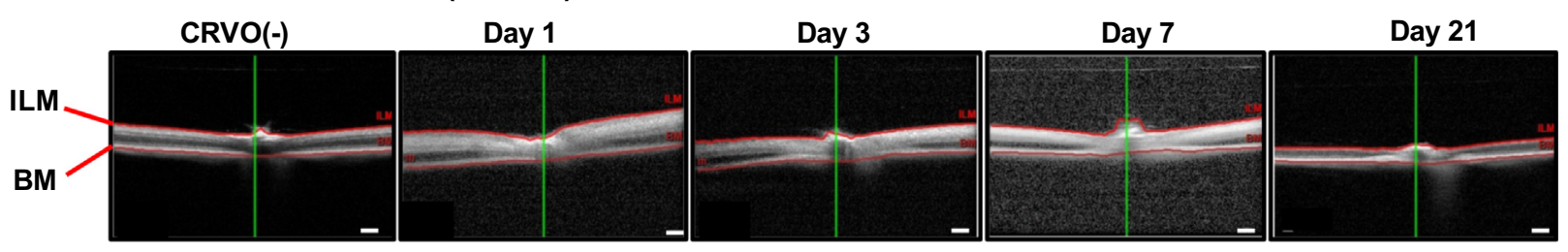

C

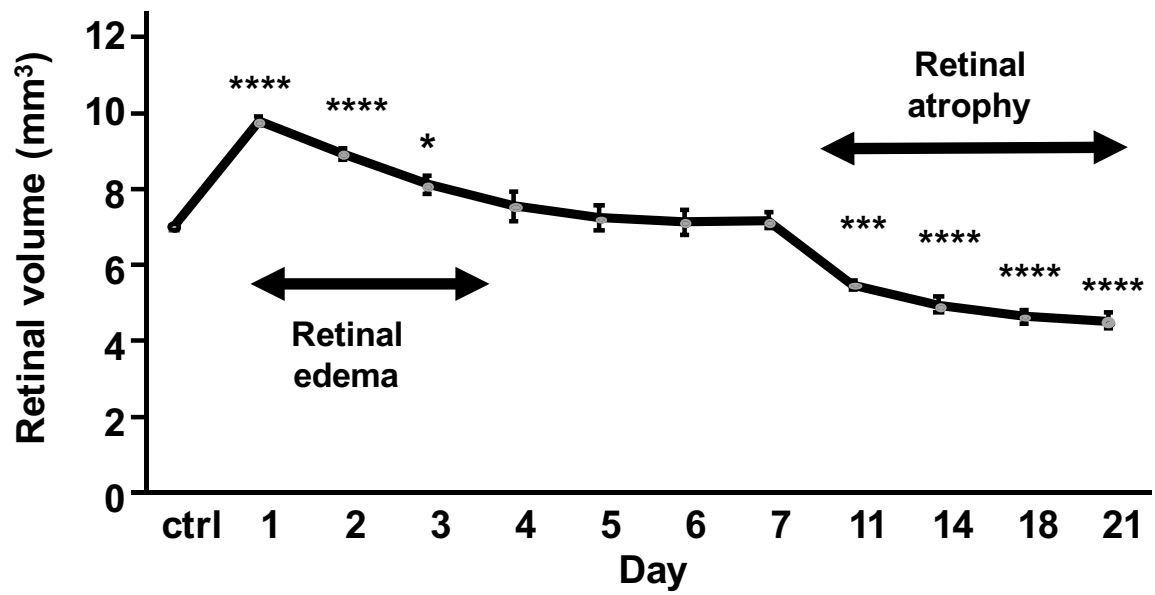

Figure 3 Evaluation of retinal volume in the central retinal vein occlusion (CRVO) model using optical coherence tomography (OCT) imaging. A: Representative OCT color mapping around the optic disc. The degree of retinal edema is shown from yellow to red, whereas retinal atrophy is shown in blue. B: B scan of OCT showing cross sections of retina between the internal limiting membrane (ILM) and the basement membrane (BM; red lines) around the optic disc. Green lines represent the center lines of retina. C: Retinal volume in a 6-mm diameter area around the optic disc calculated using the OCT algorithm. Data are expressed as means \pm SEM. $n=4$ to 6 (C). ${ }^{\star} P<0.05,{ }^{* \star} P<0.001$, and ${ }^{* \star * *} P<0.0001$ versus control (Dunnett's test). Scale bars $=200 \mu \mathrm{m}$ (B).

data were analyzed using $\mathrm{RT}^{2}$ Profiler Array Data Analysis version 3.5 software (Thermo Fisher Scientific).

\section{Quantitative Real-Time RT-PCR Analysis}

Total RNA was isolated from the retina using a PureLink RNA Mini Kit (Thermo Fisher Scientific). RNA quality was then verified using electrophoresis, and concentrations were measured using an Oubit 3.0 fluorometer (Thermo Fisher Scientific). Thereafter, the extracted RNA was treated with DNA-Free (Thermo Fisher Scientific) to remove contaminating DNA, and 2- $\mu$ g samples were subjected to reverse transcription using a PrimeScript RT reagent Kit (Takara Bio, Shiga, Japan). Quantitative real-time RT-PCR was performed using a StepOne Plus Real-Time PCR System (Thermo Fisher Scientific) with SYBR Green (Toyobo,
Osaka, Japan) or Realtime PCR Master Mix (Toyobo) and TaqMan probe (MBL, Nagoya, Japan). Values were normalized to mouse glyceraldehyde-3-phosphate dehydrogenase expression (Pre-Developed TaqMan assay reagents; Thermo Fisher Scientific) or rat glyceraldehyde-3phosphate dehydrogenase expression (using primers synthesized for rat glyceraldehyde-3-phosphate dehydrogenase expression). The primers used are listed in Table 1.

\section{Endothelial Cells}

TR-iBRB cells (FACT, Sendai, Japan), a conditionally immortalized rat retinal capillary endothelial cell line generated from rats harboring temperature-sensitive SV40 antigen, were used for this study. TR-iBRB cells were cultivated on 24-well plates in Dulbecco's modified Eagle's 
A

\section{OCT angiography}

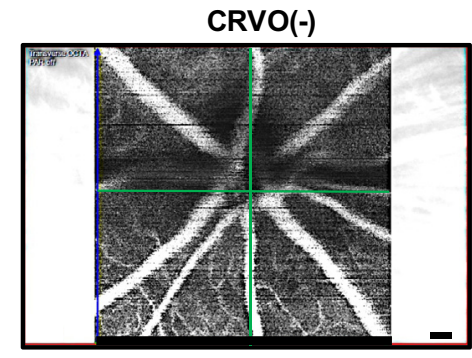

Day 7

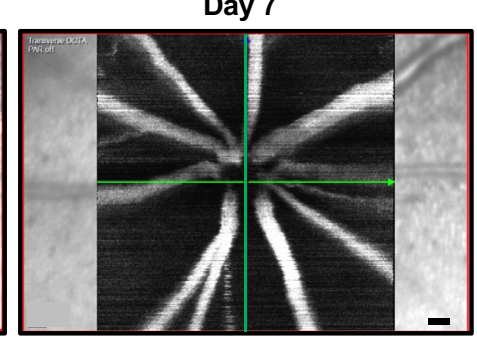

Day 21

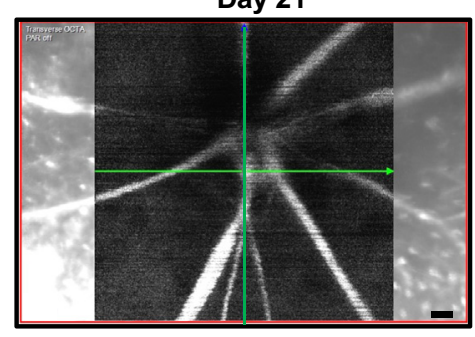

B

OCT angiography (B scan) CRVO(-)

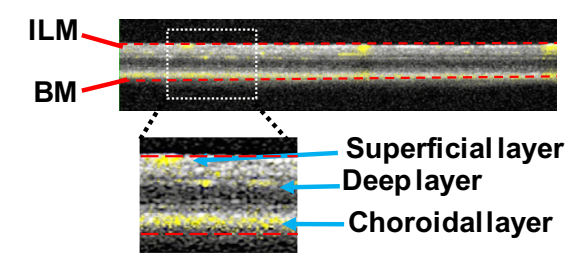

C

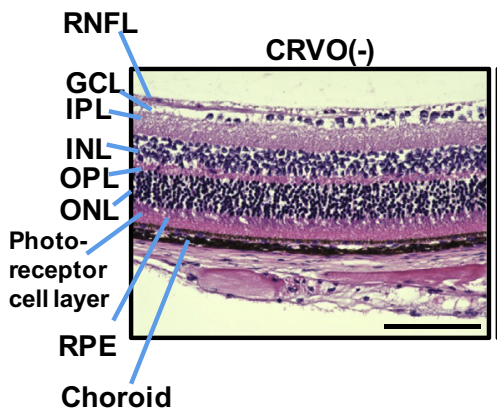

Day 7

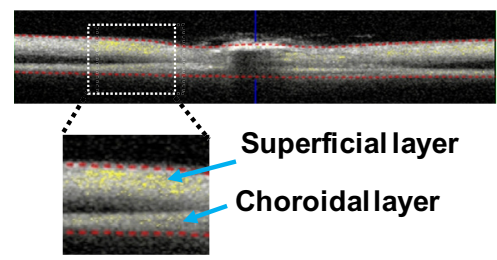

Day 21

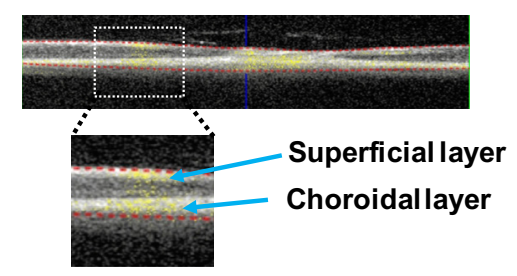

Figure 4 Evaluation of retinal blood flow based on optical coherence tomography (OCT) angiography and histologic changes in the central retinal vein occlusion (CRV0) model. A: OCT angiography with or without CRV0 treatment. Between the main retinal blood vessels radiating from the optic disc, smaller capillaries are well visualized in untreated mice. The smaller capillaries became invisible by day 7. The main retinal blood vessels are shrunken on day 21 . Green lines represent the center lines of retina. B: Top panels: B scan of OCT showing cross sections of retina between the internal limiting membrane (ILM) and the basement membrane (BM; red lines) around the optic disc. Bottom panels: Retinal layers showing blood flow signals (yellow) are enlarged. In untreated mice, blood flow signals are detected in both the superficial and deep layers, which respectively represent blood flow of the main retinal blood vessels and smaller capillaries. The blood flow in smaller capillaries in the deep layers is undetectable on days 7 and 21. C: Retinal sections with hematoxylin and eosin staining. Retinal edema was prominent from day 1 to 7 , especially at the inner layer of the retina. On day 7 , deciduation of the cells in inner nuclear layer and abnormal cavity formation within the choroid are noticeable (arrows in day 7). On day 21, the inner layers of the retina are undetectable; only the outer layers of the retina are detectable. Scale bars: $200 \mu \mathrm{m}(\mathbf{A}) ; 100 \mu \mathrm{m}(\mathbf{C}) . \mathrm{GCL}$, ganglion cell layer; INL, inner nuclear layer; IPL, inner plexiform layer; 0NL, outer nuclear layer; $\mathrm{PPL}$, outer plexiform layer; RNFL, retinal nerve fiber layer; RPE, retinal pigment epithelium.

medium supplemented with $10 \%$ fetal bovine serum and antibiotics. Once the cells reached confluence, they were stimulated for 24 hours using $20 \mathrm{ng} / \mathrm{mL}$ tumor necrosis factor- $\alpha$ with or without ADM $\left(10^{-7}\right.$ to $\left.10^{-9} \mathrm{~mol} / \mathrm{L}\right)$. Cells were then harvested and used for real-time PCR analysis.

\section{Statistical Analysis}

Statistical analysis was performed using GraphPad Prism 7.04. (GraphPad Software Inc., La Jolla, CA). Values are expressed as means \pm SEM. A $t$-test and one-way analysis of variance, followed by Dunnett's test, were used to determine the significance of differences. $P<0.05$ was considered significant.

\section{Results}

\section{Evaluation of Vascular Perfusion in the CRVO Model}

CRVO was induced using a previously described photodynamic coagulation method with some modification. ${ }^{27}$ As a sensitizing agent, Rose bengal was intraperitoneally administered. It was then confirmed that the subsequent laser radiation efficiently induced photodynamic coagulation. 
A

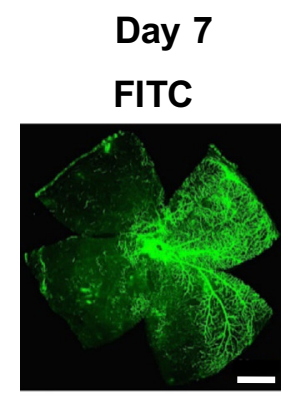

C

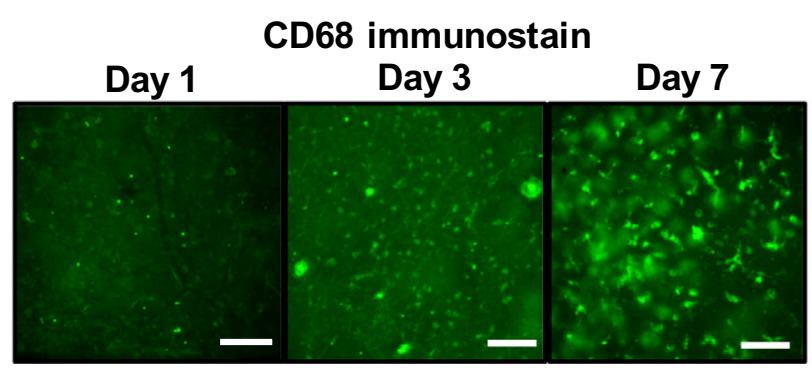

B

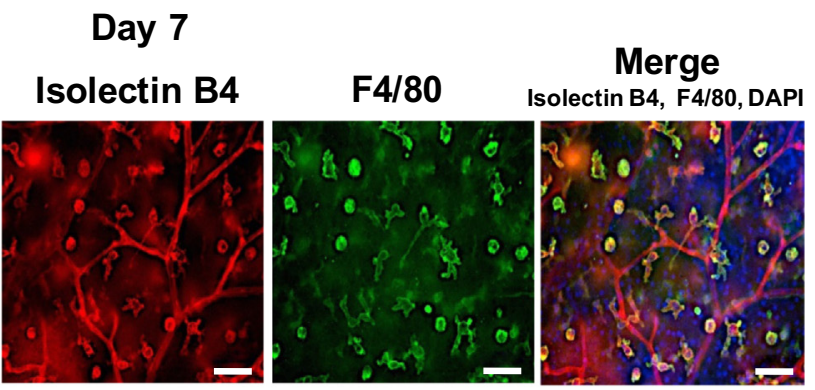

D

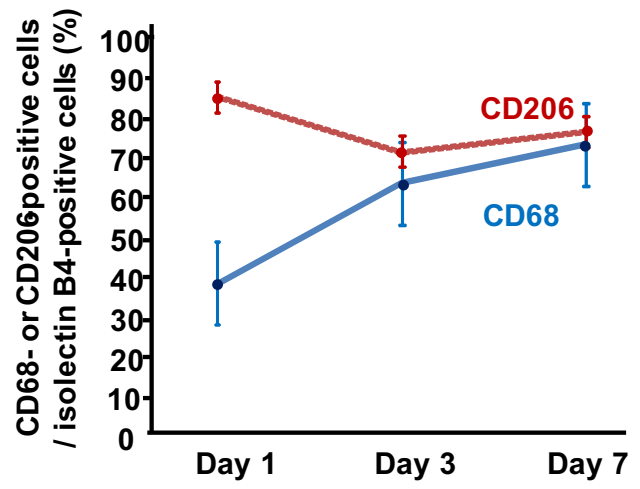

Figure 5 Evaluation of macrophages and microglia in retinal flat mounts. A: Comparison of fluorescein isothiocyanate (FITC)-perfused and isolectin B4-stained retinal flat mounts on day 7 after central retinal vein occlusion (CRV0). The distribution of FITC-positive and isolectin B4-positive areas is strikingly different. With FITC perfusion, nonperfused areas are not clearly seen, whereas isolectin B4-stained areas are more likely to be detected in nonperfused areas. B: Comparison of isolectin B4 staining and F4/80 immunostaining of retinal flat mounts on day 7 after CRV0. Left panel: Isolectin B4 staining is detected on small round structures with dendrites, which are clearly different from the vascular branch structures. Middle panel: These structures are also positive for the macrophage marker F4/80, showing that macrophage numbers are increased in nonperfused areas after CRV0. Right panel: Merge of isolectin F4/80, an DAPI. C: CD68 immunostaining of retinal flat mounts after CRV0. CD68-positive macrophages and activated microglia increase over time from day 1 to day 7. D: Percentage of CD68- or CD206-positive cells among isolectin B4-positive cells in retinal flat mounts after CRV0 (isolectin B4-positive vessels were excluded from the calculation). CD68-positve cells are rarely observed before CRV0 but increase after CRV0. Data are expressed as means \pm SEM. $n=5$ in each group (D). Scale bars: $100 \mu \mathrm{m}$ (A and C); $50 \mu \mathrm{m}$ (B).

In fundus images acquired on day 7 , retinal hemorrhage as well as retinal vein dilatation with tortuousness were observed in the treated mice (Figure 1A). Retinal flat-mount images also showed prominent hemorrhage in the treated mice (Figure 1B). Retinal hemorrhage is the most characteristic finding of clinical CRVO and is not observed in central retinal artery occlusion. This confirms that a CRVO model was successfully generated.

FA was performed on days 1, 3, and 7 after CRVO. After injecting FITC via the heart, retinal flat mounts were prepared. FA revealed the percentage vascular area of the retina to be $26 \%$ in untreated mice. On day 1 after CRVO, lack of vascular perfusion due to blood vessel occlusion was prominent, and the percentage vascular area was only $2.7 \%$. Thereafter, vascular perfusion gradually recovered over time, but the percentage vascular retinal area remained lower than control on day 7 (day 3, 8.4\%; day 7, 17\%) (Figure 2, A and B). FITC-perfused retinal flat mounts also showed decreased vascular area on day 7 (untreated group, 53\%; CRVO group, 30\%) (Figure 2, C and D).

\section{Evaluation of Retinal Edema in the CRVO Model Using OCT and Histology}

OCT was used to evaluate post-CRVO retinal edema. With color mapping using clinical OCT equipment (Heidelberg SPECTRALIS OCT), the degree of retinal edema and retinal atrophy was studied (Figure 3A). On OCT B scans, cross sections of the retina between the internal limiting membrane and basement membrane were visualized (Figure 3B). The retinal volume within a region $6 \mathrm{~mm}$ in diameter around the optic disc was automatically calculated by the algorithm built into the OCT equipment (Figure 3C).

The retinal volume was $7.0 \mathrm{~mm}^{3}$ in the no treatment group. Retinal edema was most prominent on day 1, and retinal volume reached 1.4 times the baseline level. Because of the edema, the structure of the inner layer of the retina became ambiguous on the B scan. Thereafter, the retinal volume gradually declined to the baseline level by day $7\left(7.2 \mathrm{~mm}^{3}\right)$. After day 11, however, the retinal volume continued to decline to levels significantly lower than baseline. This retinal atrophy 


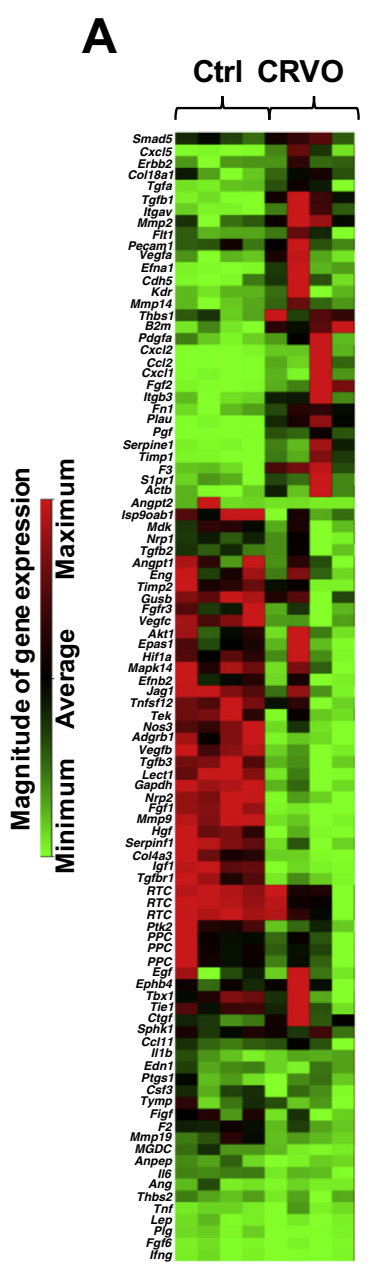

B

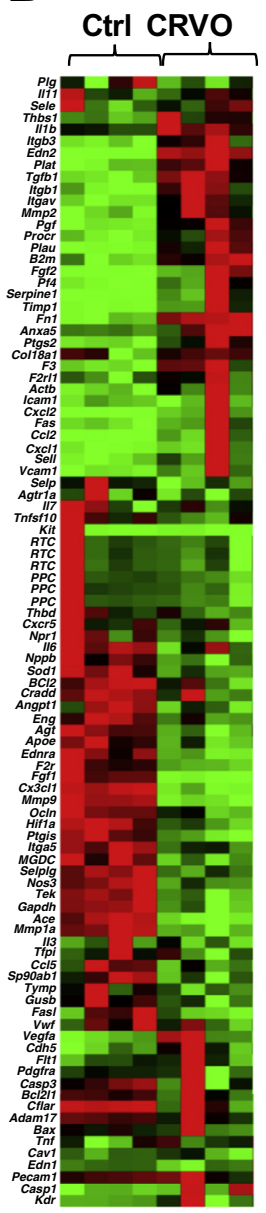

C
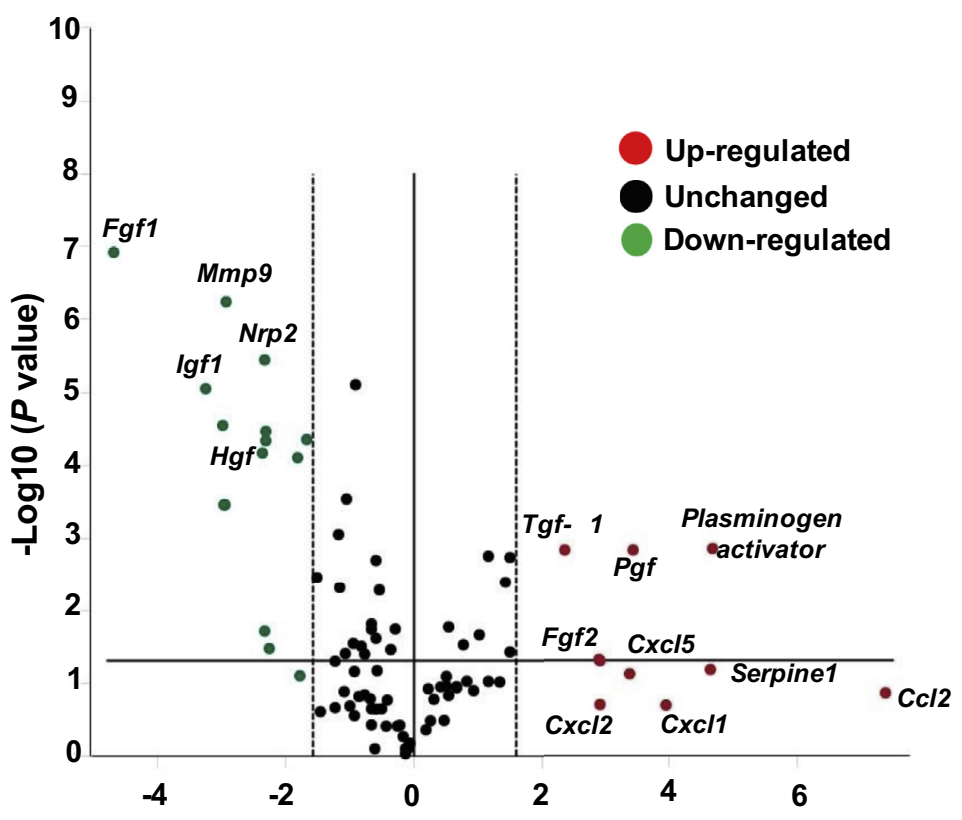

Log2 (fold change: CRVO treat/no treat)

Figure 6 Comprehensive gene expression analysis in retinas with and without central retinal vein occlusion (CRV0). A and B: Clustergram showing the results of real-time PCR array analysis of angiogenesis-related (A) and endothelial cell-related (B) molecules in mouse retinas with and without CRVO treatment. Each column shows the results from one mouse. Red and green colors represent higher and lower gene expression, respectively. C: Dot plot showing the results of the real-time PCR array analysis. The horizontal axis shows the fold change $\left[\log _{2}\right.$ (fold change)], and the vertical axis shows the $P$ value $\left[-\log _{10}\right.$ $(P$ value)]. Dotted lines indicate the distribution of unchanged genes. In CRV0-treated mice, expression of chemokines, growth factors, and coagulation factors is up-regulated compared with untreated mice. Some angiogenic factors are down-regulated in the vascular occlusion group. Ctrl, control.

continued until day $21\left(4.2 \mathrm{~mm}^{3}\right)$. The retinal thickness was also analyzed using OCT equipment for smaller experimental animals (Envisu R-Class OCT), and similar results were obtained (Supplemental Figures S1 and S2).

Using OCT angiography, smaller capillaries were observed between the main retinal blood vessels radiating from the optic disc (arteries and veins) in the untreated mice (Figure 4A). These smaller capillaries were undetectable on day 7 after CRVO, however, although the main retinal blood vessels were still well visualized at this stage (Figure 4A). In B scans of the OCT angiography in untreated mice, blood flow signals were detected at both superficial and deep layers, which respectively represent blood flow in the main retinal blood vessels and smaller capillaries (Figure 4B). The blood flow in the smaller capillaries in the deep layer was undetectable on day 7 after CRVO (Figure 4B). These observations indicate that small capillary dropout is irreversible, although the main retinal blood vessels show reperfusion on day 7 after CRVO. By day 21 after CRVO, however, the main retinal blood vessels appeared shrunken (Figure 4A).

Histologic changes to the retinal layer structure were examined in hematoxylin and eosin-stained cross sections prepared from retinas with and without CRVO (days 1, 7, and 21) (Figure 4C). Retinal edema was prominent, especially at the inner layer of the retina from day 1 to day 7. On day 7, deciduation of the cells in the inner nuclear layer and abnormal cavity formation within the choroid were noticeable. On day 21 , the inner retinal layer was undetectable, and only outer layers of the retina (outer plexiform layer and outer nuclear layer) were detectable. These histologic observations of retinal edema and atrophy correspond well to the OCT analysis.

\section{Evaluation of Macrophages and Microglia in Retinal Flat Mounts}

Isolectin B4 staining has been previously used to visualize endothelial cells in the retinal vasculature. ${ }^{25}$ Comparing 

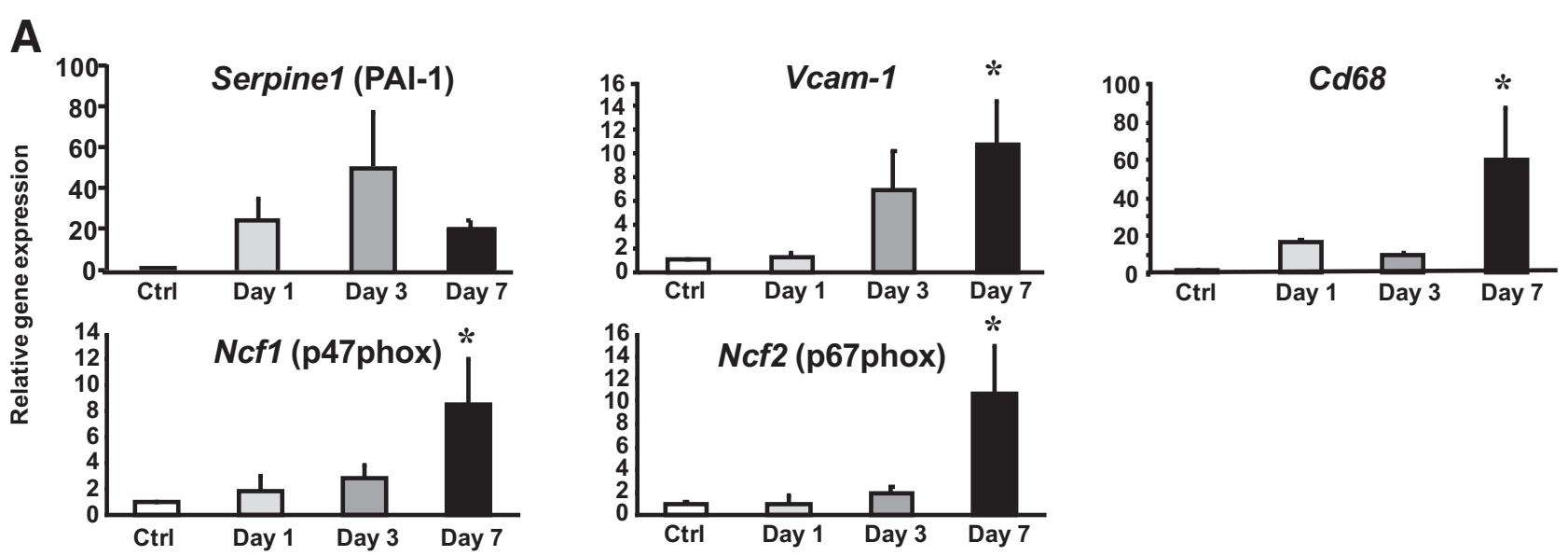

B
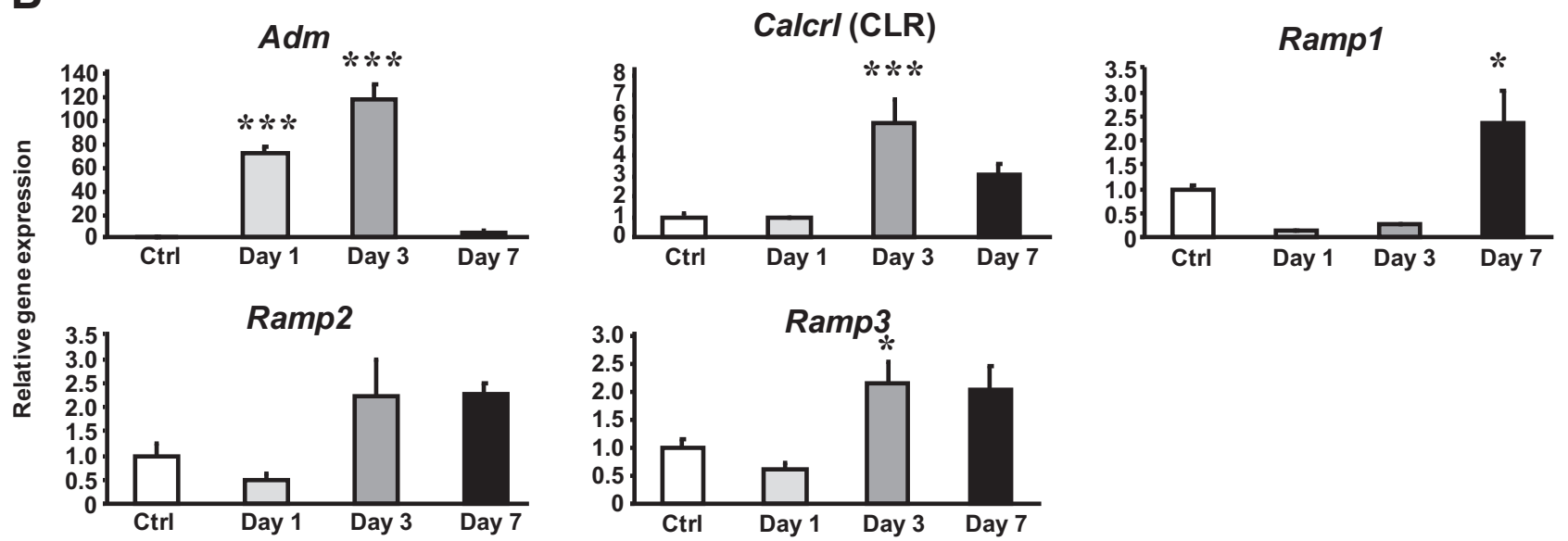

Figure 7 Quantitative real-time PCR analysis of retinas with or without central retinal vein occlusion (CRV0). A: Relative expression of coagulation-, inflammation-, and oxidative stress-related genes in the retinas of untreated mice and on days 1, 3, and 7 after CRV0. The mean of the control group without CRV0 treatment (Ctrl) was assigned a value of 1. B: Relative expression of Adm and its related genes in retinas of untreated mice and on days 1, 3, and 7 after CRV0. Data are expressed as means \pm SEM. $n=4$ in each group (A and B). ${ }^{*} P<0.05,{ }^{* *} P<0.001$ versus control (Dunnett's test). CLR, calcitonin receptor-like receptor; PAI, plasminogen activator inhibitor.

images of FITC perfusion and isolectin B4 staining in retinal flat mounts after CRVO, the distributions of FITC and isolectin B4 positivity were found to differ somewhat (Figure 5A). In images of FITC-perfused retinas, nonperfused areas could not be clearly visualized (Figure 5A). On the other hand, isolectin B4-stained areas were detected in both perfused and nonperfused areas. Isolectin B4 positivity without FITC perfusion may be indicative of nonperfused vessels. Moreover, isolectin B4 staining was much stronger in nonperfused than perfused areas (Figure 5A). Under higher magnification of nonperfused areas, isolectin B4 positivity was also observed in small round structures that were clearly different from vascular branch structures (Figure 5B), and some had dendrites. The round structures also stained positive for the macrophage marker F4/80 (Figure 5B). Thus, macrophage numbers were increased in the nonperfused areas after induction of CRVO. This suggests inflammation is more severe in the nonperfused ischemic areas of the retina.
CD206 is a marker of M2 macrophages, whereas CD68 is a marker of macrophages and activated microglia. ${ }^{29}$ CD206positive cells were mainly located in the inner layer of retina and are thought to be resident microglia. Percentages of CD206-positive microglia declined slightly after CRVO (Figure 5D). By contrast, CD68-positve cells were rarely observed before CRVO, but after CRVO their numbers increased over time, and they were distributed in all layers of the retina (Figure 5, C and D). CD68-positive cells were therefore thought to be either proinflammatory macrophages derived from outside of the retina or activated microglia, both of which are thought to be involved in retinal inflammation after CRVO.

\section{Evaluation of the Gene Expression with and without CRVO}

To further evaluate the pathogenesis of the CRVO model, a real-time PCR array analysis of angiogenesis- and 
A

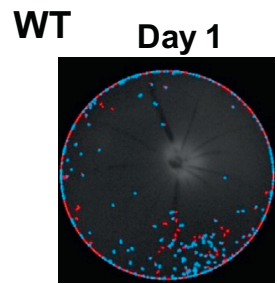

ADM KO

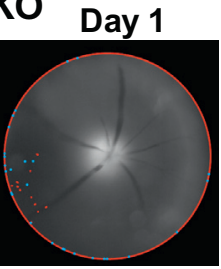

Day 1

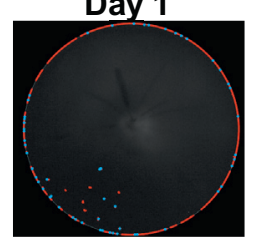

Day 3
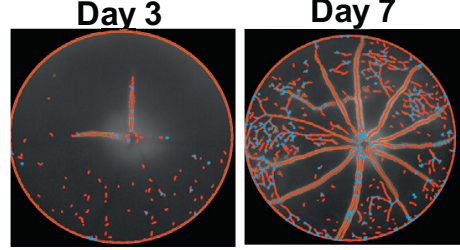

Day 3

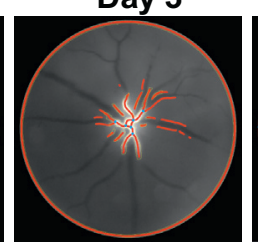

Day 7

RAMP2 KO

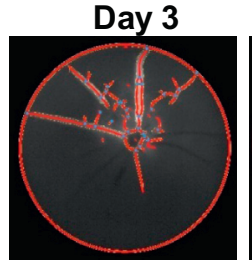

B
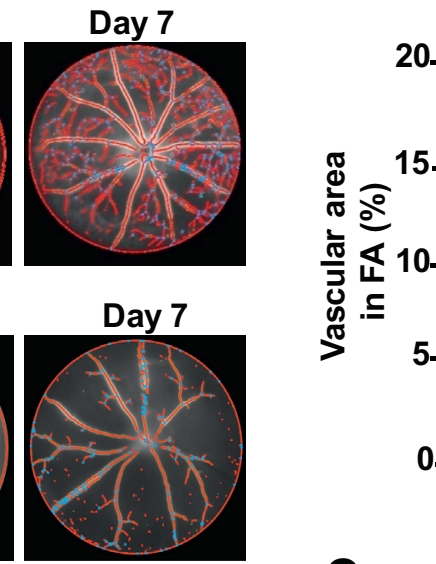

20

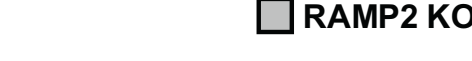

C

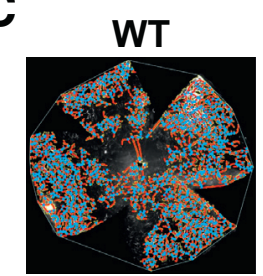

WT

ADM KO

RAMP2 KO

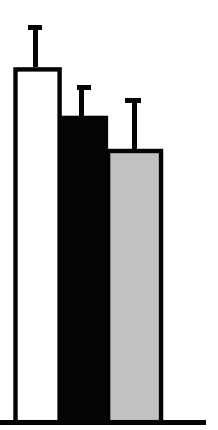

Day 3

Day 7
RAMP2 KO
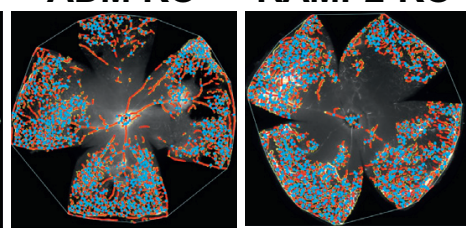

D

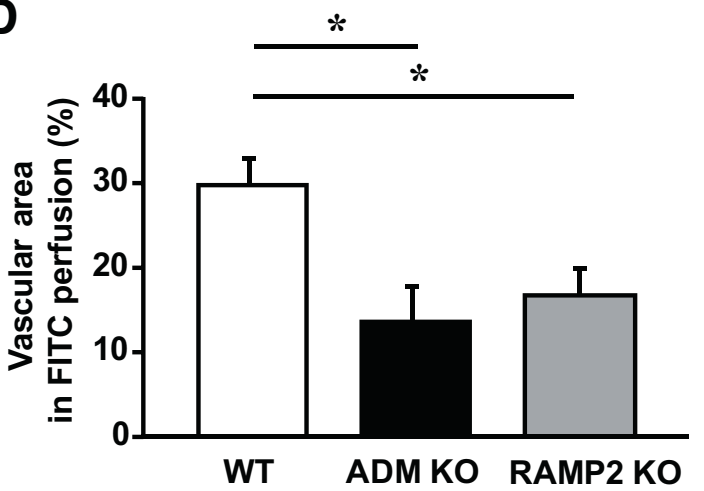

Figure $8 \quad A d m$ and Ramp2 knockout (KO) mice show reduced vascular perfusion after central retinal vein occlusion (CRV0). A: Fluorescein angiography (FA) images analyzed using AngioTool software version 0.6a in wild-type (WT), Adm K0, and Ramp2 K0 mice after CRV0 treatment (days 1, 3, and 7). Blood vessels are depicted in red, and vascular branch points are depicted in blue. B: Percentage vascular area in FA images quantified using AngioTool. C: Fluorescein isothiocyanate (FITC)perfused retinal flat-mount images analyzed using AngioTool. Blood vessels are depicted in red, and vascular branch points are depicted in blue. D: Percentage vascular area in FITC-perfused retinal flat mounts quantified using AngioTool. Data are expressed as means \pm SEM. $n=5$ to 8 (B); $n=5$ to 7 (D). ${ }^{*} P<0.05$ (Dunnett's test). endothelial cell-related molecules was performed in mouse retinas with and without CRVO. All the examined mice in each group showed similar gene expression profiles (Figure 6, A and B). After induction of CRVO, expression levels of chemokines, growth factors, and coagulation factors were higher than in the untreated group (Figure 6C). On the other hand, some angiogenic factors, including Fgfl, $M m p 9$, Nrp2, Igfl, and $H g f$, were down-regulated in the vascular occlusion group (Figure 6C).

Analysis of the time course of the gene expression revealed that the coagulation-related molecule Serpine1 [plasminogen activator inhibitor 1 (PAI-1)] was most prominently upregulated on day 3 after CRVO (52-fold) (Figure 7A). A leukocyte adhesion molecule, Vcaml, was prominently upregulated (11-fold) on day 7 after CRVO. In association with the inflammatory reaction, the NADPH subunits Ncfl (p47phox) and $N c f 2$ (p67phox) were also up-regulated on day 7 (Ncf1, 8.5-fold; Ncf2, 11-fold), as was the macrophage and activated microglia marker $C d 68$ (60-fold). These observations suggest enhanced coagulation, inflammation, and oxidative stress are all involved in the pathogenesis of CRVO. Adm expression was significantly up-regulated on days 1 (72-fold) and 3 (118-fold) after CRVO, but it had declined by day 7 (4.5fold) (Figure 7B). Adm's receptor, Calcrl (CLR), was most upregulated on day 3 (5.6-fold), whereas Ramps were upregulated on days 3 and 7 . The timing of the dynamic changes in gene expression suggests ADM and its receptor system are crucially involved in the pathogenesis of CRVO.

\section{Adm and Ramp2 Knockout Mice Show More Severe Phenotypes in the CRVO Model}

To evaluate the role played by ADM in the pathophysiology of CRVO, heterozygotic Adm and Ramp2 KO mice were compared with WT mice. In Adm KO mice, serum ADM was reduced to $70 \%$ of the level in wild-type mice 
A

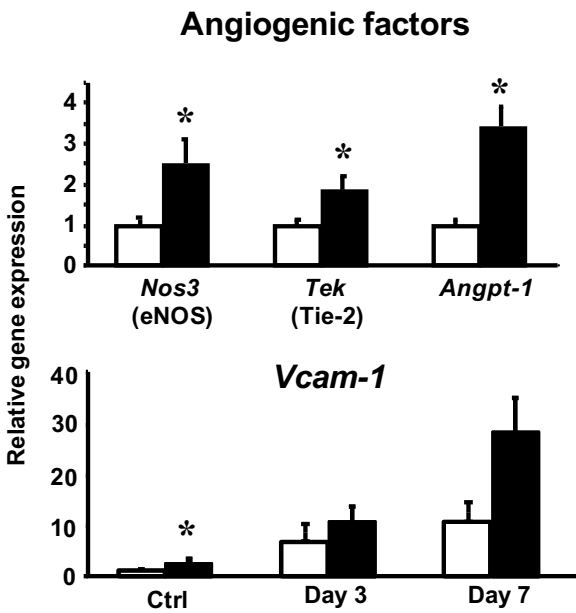

Serpine1 (PAI-1)
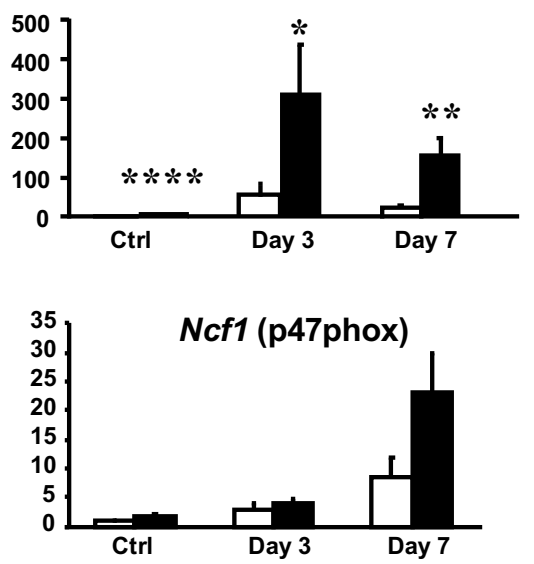

Cd68 $\square$ ADM KO
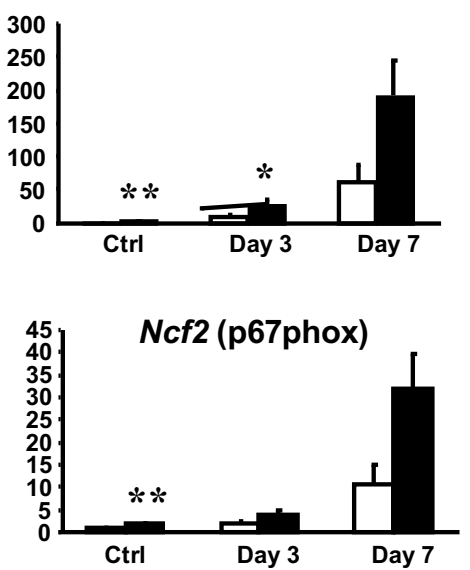

B CD68-positive cells
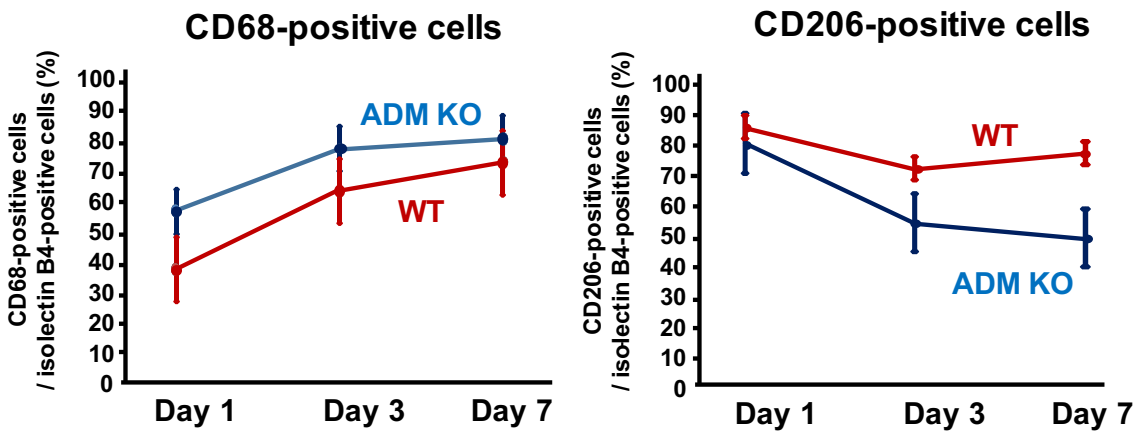

Figure 9 Gene expression and macrophage distribution in the retinas of Adm knockout (KO) mice. A: Quantitative real-time PCR analysis showing the relative expression levels of the indicated genes in the retinas of $A d m$ KO and wild-type (WT) mice. The mean in WT mice was assigned a value of 1 . B: Percentage of CD68- or CD206-positive cells among isolectin B4-positive cells in retinal flat mounts after central retinal vein occlusion. Percentages of CD68positive macrophages and activated microglia are increased, whereas CD206-positive resident microglia are decreased, in Adm K0 compared with WT mice. Data are expressed as means \pm SEM. $n=4$ in each group (A); $n=8$ to 10 in each group (B). ${ }^{*} P<0.05,{ }^{* *} P<0.01$, and ${ }^{* * * *} P<0.0001$ versus WT (unpaired $t$-test). Ctrl, control; eNOS, endothelial nitric oxide synthase; PAI, plasminogen activator inhibitor.

(Supplemental Figure S3). FA showed that after CRVO in WT mice, the percentage vascular area of the retina was initially decreased but gradually recovered from day 1 to 7 (Figure 8, A and B). In Adm and Ramp2 KO mice, there tended to be less recovery than in WT mice (Adm KO: day 1, $1.3 \%$; day $3,5.9 \%$; day $7,15 \%$; and Ramp 2 KO: day 1 , $0.75 \%$; day 3, 3.0\%; day 7, 14\%). In addition, FITCperfused retinal flat mounts (Figure 8, C and D) showed significantly less vascular area in $A d m \mathrm{KO}(13.0 \%)$ and Ramp2 KO (17.0\%) mice than in WT mice (30\%).

Comparison of the retinal gene expression profiles of WT and $A d m \mathrm{KO}$ with and without CRVO revealed that the angiogenic factors Nos3 (endothelial nitric oxide synthase), Tek (Tie-2; tyrosine kinase with IgG and EGF homology domain), and Angptl were significantly up-regulated in $\mathrm{Adm}$ $\mathrm{KO}$ at baseline (Figure 9A). Because ADM has angiogenic properties, up-regulation of these molecules is thought to be a compensatory response to the reduction in ADM. Levels of these angiogenic molecules also tended to be higher in Adm KO after CRVO (data not shown).

Comparison of Serpine1 (PAI-1), Cd68, Vcam1, Ncfl (p47phox), and $N c f 2$ (p67phox) expression between WT and $A d m \mathrm{KO}$ mice showed that, even before CRVO, levels of Serpine1, Cd68, Vcam1, and Ncf2 expression were significantly higher in $A d m$ KO than WT mice (Figure 9A). Expression of $N c f l$ also tended to be elevated in $A d m \mathrm{KO}$ mice. As mentioned above, the expression of these genes was up-regulated after CRVO, but the effect was much more pronounced in $A d m \mathrm{KO}$ mice. In retinal flat mounts, the numbers of CD68-positive macrophages and activated microglia were higher in Adm KO than WT mice, whereas the numbers of CD206-positive resident microglia were lower (Figure 9B). These observations suggest that reducing $A d m$ expression exacerbates inflammation and suppresses vascular reperfusion after CRVO. Thus, endogenous ADM likely exerts protective effects against the retinal damage caused by CRVO.

ADM Administration Ameliorates the Phenotypes in the CRVO Model

The therapeutic effects of exogenous ADM administration were next evaluated in the CRVO model. ADM was systemically administered using s.c. osmotic pumps. In 

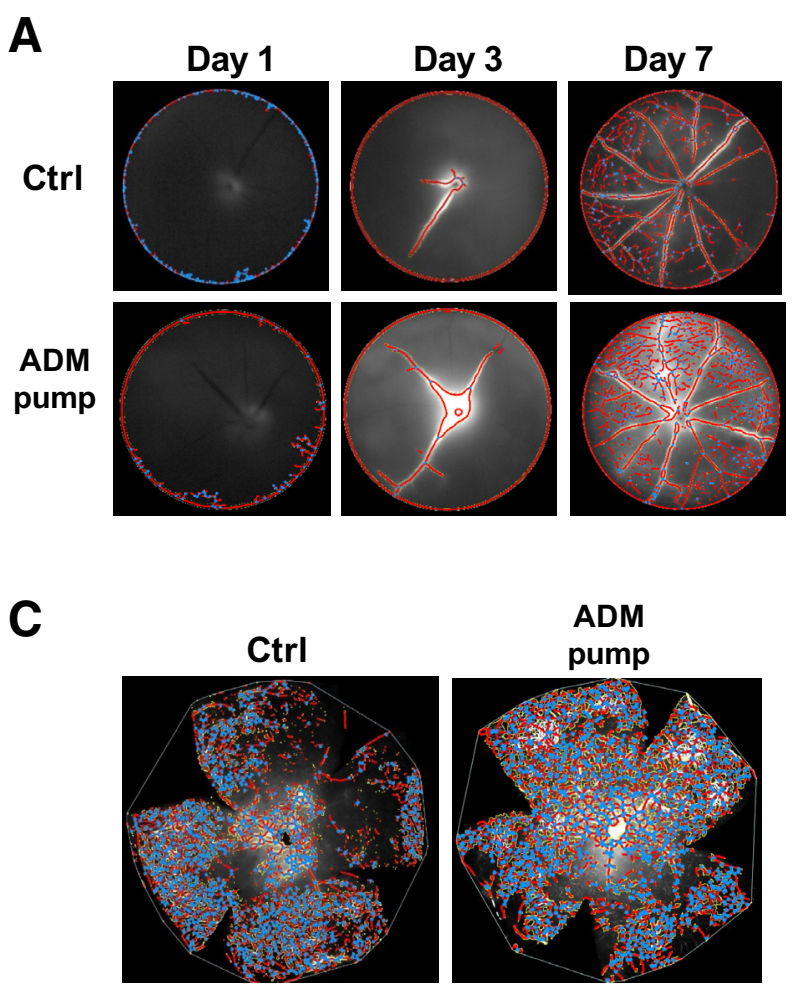

B
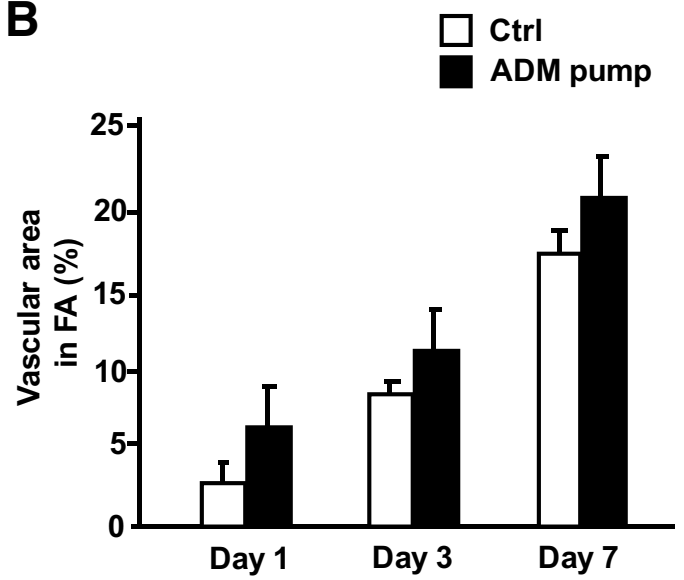

D

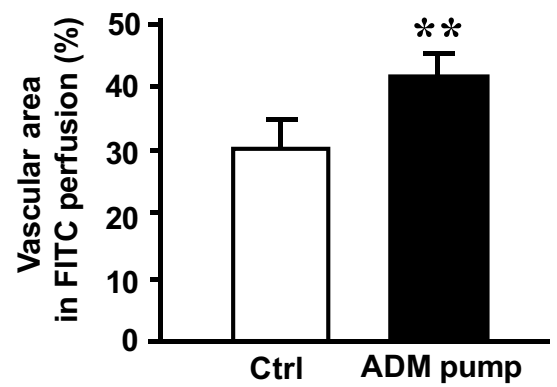

Figure 10 Adrenomedullin (ADM) administration improves vascular perfusion after central retinal vein occlusion (CRVO). A: Fluorescein angiography (FA) images of retinas analyzed using AngioTool software version 0.6a in control wild-type (WT) mice [control (Ctrl)] and WT mice administered ADM (ADM pump) on the indicated days after CRVO. Blood vessels are depicted in red, and vascular branch points are depicted in blue. B: Percentage vascular area in the FA images was quantified using AngioTool software. C: Fluorescein isothiocyanate (FITC)-perfused retinal flat mounts analyzed using AngioTool. Blood vessels are depicted in red, and vascular branch points are depicted in blue. D: Percentage vascular area in FITC-perfused retinal flat mounts was quantified using AngioTool. ADM administration improves vascular perfusion. Data are expressed as means \pm SEM. $n=4$ to 9 (B); $n=5$ in each group (D). ${ }^{* *} P<0.01$ versus control (unpaired $t$-test).

ADM-administered mice, serum ADM was 50\% higher than in wild-type mice (Supplemental Figure S3). Both FA- and FITC-perfused retinal flat mounts showed that vascular reperfusion after CRVO was increased by ADM administration (Figure 10), whereas FITC-perfused flat mounts showed that ADM significantly increased vascular area (control, 30\%; ADM administration, 45\%). In a real-time PCR array analysis, ADM administration up-regulated several angiogenic factors and down-regulated inflammation-related cytokines and chemokines (Supplemental Figure S4). ADM administration markedly suppressed expression of Serpinel (PAI-1), Cd68, Vcaml, Ncfl (p47phox), and $N c f 2$ (p67phox) (Figure 11A). In retinal flat mounts, numbers of CD68-positive macrophages and activated microglia were decreased and CD206-positive resident microglia were increased after ADM administration (Figure 11B). In addition, expression of $K d r$ (VEGF receptor-2) was greatly elevated after CRVO (day 3, 7.9fold; day 7, 9.8-fold), and that effect too was markedly suppressed by ADM administration.

The cross-sectional histology of the retinal layers was also analyzed (Figure 12). On day 7 after CRVO, disruption of the inner retinal layer was exacerbated in $A d m \mathrm{KO}$ mice and suppressed by ADM administration. Moreover, Adm KO mice showed elevated expression of $N c f 2$ (p67phox), which is indicative of a higher level of oxidative stress. These observations suggest ADM administration ameliorates the pathology of CRVO by enhancing vascular reperfusion and suppressing both inflammation and oxidative stress.

\section{ADM Directly Suppresses Retinal Endothelial Injury}

Given the effects of ADM summarized above, we speculated that ADM suppresses vascular injury after CRVO. To test that idea, the effect of ADM administration on TR-iBRB cells, an immortalized retinal capillary endothelial cell line, was assessed. To induce endothelial injury, the cells were stimulated for 24 hours with $20 \mathrm{ng} / \mathrm{mL}$ tumor necrosis factor$\alpha$ in the presence or absence of ADM $\left(10^{-7}\right.$ to $\left.10^{-9} \mathrm{~mol} / \mathrm{L}\right)$. Subsequent quantitative real-time PCR analysis showed that expression of inflammatory and oxidative stress-related molecules [Vcaml, Ccl2 (MCP-1; monocyte chemotactic protein 1), Cd68, Ncf1 (p47phox), and Ncf2 (p67phox)] was highly up-regulated by tumor necrosis factor- $\alpha$ administration, but coadministration of ADM down-regulated that 
A
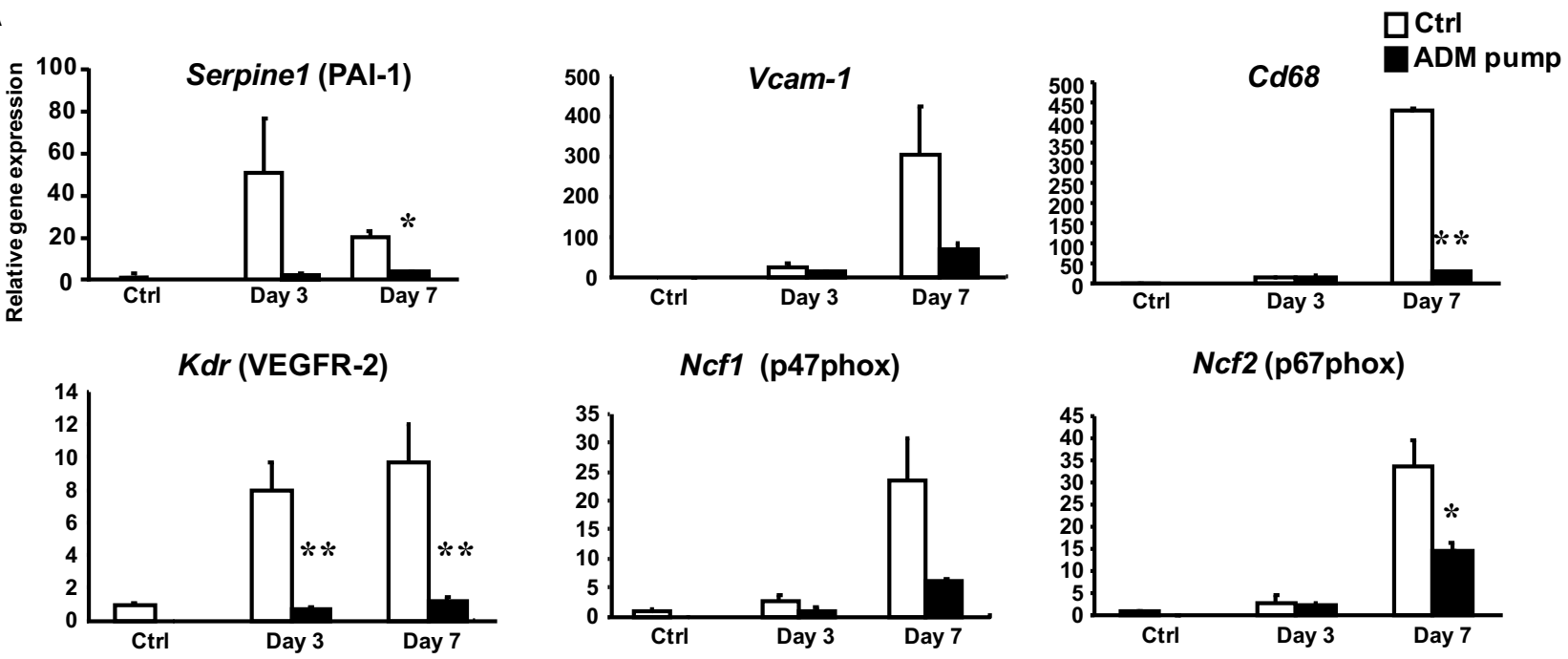

B
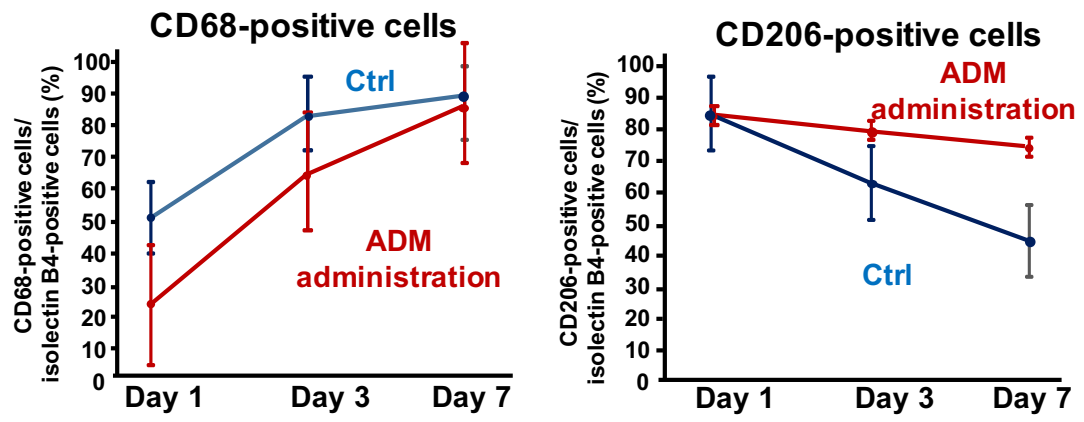

Figure 11 Gene expression and macrophage distribution in the retinas of mice administered adrenomedullin (ADM). A: Quantitative real-time PCR analysis showing the relative expression of the indicated genes in the retinas of control wild-type (WT) mice (Ctrl) and WT mice administered ADM (ADM pump). The mean of the Ctrl group was assigned a value of 1. B: Percentage of CD68- or CD206-positive cells among isolectin B4-positive cells in retinal flat mounts after central retinal vein occlusion. Percentages of CD68-positive macrophages and activated microglia are decreased, whereas CD206-positive resident microglia are increased, by intravitreal injection of ADM. Data are expressed as means \pm SEM. $n=4$ in each group $(\mathbf{A}) ; n=5$ in each group $(\mathbf{B})$. ${ }^{\star} P<0.05,{ }^{*} P<0.01$ versus control (unpaired $t$-test). PAI, plasminogen activator inhibitor.

expression (Figure 13). ADM administration also downregulated expression of $K d r$ (VEGF receptor-2). These observations corresponded well to the results obtained with the CRVO model and suggest ADM exerts direct protective effects against retinal endothelial cell injury.

\section{Discussion}

RVO affects an estimated 16.4 million individuals worldwide and is one of the most common retinal vascular diseases in adults, second only to DR. ${ }^{1}$ In CRVO, venous dilatation with tortuousness, hemorrhage, and edema occurs in association with venous occlusion. There are two types of CRVO, ischemic and nonischemic, ${ }^{30}$ with the ischemic type being refractory to treatment. ${ }^{31}$ Capillary dropout is a key feature of ischemic RVO, and when it occurs in the vicinity of the fovea, there is irreversible loss of visual acuity.

The main purpose for the current clinical use of antiVEGF drugs against CRVO is to suppress excessive enhancement of retinal vascular permeability; it is not to inhibit angiogenesis. VEGF enhances vascular permeability, and anti-VEGF drugs are able to mitigate the resultant macular edema. However, the beneficial effect of antiVEGF drugs on visual acuity is not permanent, ${ }^{32}$ and the need to periodically reinject the drug increases the risk of eye infection. ${ }^{33}$ In addition, the use of large amounts of antibody increases medical expenses. For those reasons, reducing the number of injections would be beneficial from both therapeutic and financial viewpoints. Furthermore, the use of anti-VEGF drugs reportedly exacerbates retinal geographic atrophy. ${ }^{34}$ Moreover, because VEGF almost certainly has a physiological function in the eye, we would expect that chronic inhibition of VEGF signaling would have adverse effects.

Hypertension, hyperlipidemia, diabetes, and aging are all risk factors of CRVO. Vascular dysfunction associated with these risk factors leads to stagnation of venous blood flow within the retina, which may lead to the onset of CRVO. ${ }^{35}$ At present, however, the methods available for vascular treatment are limited, and the search for novel therapeutic targets is an urgent issue. 


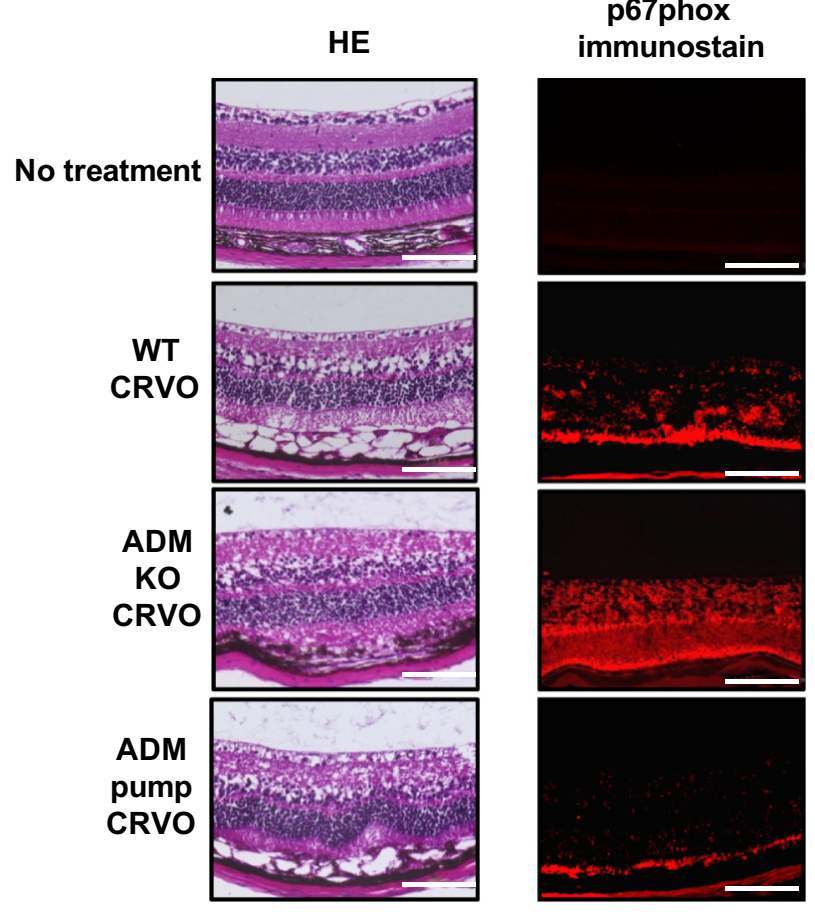

Figure 12 Retinal damage is enhanced in $A d m$ knockout (KO) and suppressed by adrenomedullin (ADM) administration. Hematoxylin and eosin (HE) staining and p67phox immunostaining in cross sections showing the retinal layers on day 7 after central retinal vein occlusion (CRVO). Disruption of the internal retinal layer is exacerbated in $A d m \mathrm{KO}$ mice and is suppressed in the mice administered ADM (ADM pump). Adm KO mice also show elevated p67phox expression, indicating higher oxidative stress. Scale bars $=100 \mu \mathrm{m}$. WT, wild type.

Up to now, there have been no reports of a CRVO model in mice, except for one accidentally obtained during an attempt to generate a branch retinal vein occlusion model. ${ }^{36}$ By combining i.p. injection of Rose bengal with laser irradiation, a mouse model exhibiting phenotypes similar to those of clinical CRVO was generated. To achieve an efficient photodynamic thrombotic effect, the sensitizing agent (Rose bengal) was exposed to focused light at its peak absorption wavelength to generate oxygen singlets. ${ }^{37}$ Within blood vessels, the singlet oxygen induces damage to endothelial cell membranes, which likely serves as the initial stimulus for platelet adhesion and aggregation, leading to vascular occlusion. In the earlier report on the branch retinal vein occlusion model, Rose bengal was injected via the tail vein. ${ }^{27}$ However, tail vein injection is difficult in the mouse, and the procedure requires more practice than i.p. injection, which is easy to perform. And because there are few failures with i.p. injection, multiple samples can be handled in a single experiment.

Another advantage of the i.p. injection of Rose bengal is that the appropriate blood concentration is readily achieved. In an earlier report on central retinal artery occlusion model mice, Rose bengal was injected via the tail vein, ${ }^{38}$ but retinal hemorrhage, which is characteristic of CRVO, was not detected. This may have been due to extensive clogging of the blood vessels. By contrast, with the lower blood concentration of Rose bengal achieved with i.p. injection, retinal arteries could be reopened in our model. This model, therefore, shows resemblance to clinical CRVO, with retinal hemorrhage and vein dilatation with tortuousness.

A limitation of this model is that retinal artery occlusion using laser photocoagulation is accompanied by venous occlusion. In a strict sense, therefore, this model does not completely reproduce clinical human CRVO. However, using the experimental CRVO model, it was previously shown that, by itself, venous occlusion does not cause retinal hemorrhage. ${ }^{39}$ Retinal hemorrhage is the most characteristic phenotype of CRVO and is not observed in central retinal artery occlusion. ${ }^{40}$ It is caused by extravasation of blood due to venous occlusion. In this model, retinal hemorrhage typical of CRVO was observed. In the past experimental studies, typical hemorrhage was observed only when both the vein and artery were occluded. ${ }^{41,42}$ This suggests that when researchers want to produce an experimental model of CRVO, they must also transiently occlude the artery. In addition, in the clinical situation, CRVO and central retinal artery occlusion are sometimes observed together in the same patient. ${ }^{43}$ We therefore think that our model reproduces key aspects of clinical CRVO.

In this model, lack of perfusion due to blood vessel occlusion was most prominent on day 1. After that, vascular perfusion gradually improved over time, although it had not fully recovered by day 7 . Because leukocyte stagnation within capillary blood vessels promotes capillary dropout, ${ }^{44}$ maintaining blood vessel density with effective blood flow and suppression of leukocyte adhesion after disease onset is considered to be important for effective treatment of CRVO. Compared with WT mice, Adm and Ramp2 KO mice showed less blood flow recovery on day 7 . On the other hand, vascular reperfusion after CRVO was enhanced through exogenous ADM administration. We therefore suggest that ADM may be useful in suppressing capillary dropout and improving the condition.

CRVO can also cause vision loss through secondary macular edema. Early relief of edema after the onset of CRVO is important for visual prognosis. ${ }^{45}$ This study is the first to evaluate retinal volume in mice using OCT. In our model, as in human patients, retinal edema occurred after CRVO onset. The retinal edema gradually declined and had reached baseline levels by day 7 . Retinal edema was also exacerbated in Adm KO mice, and that effect was suppressed by ADM administration.

Quantitative real-time PCR analysis showed that the expression of $A d m$ and its receptor, Calcrl (CLR), was increased by as much as 120- and 6-fold, respectively, on day 3 after the CRVO. This marked up-regulation of $A d m$ and its receptor strongly suggests the involvement of ADM signaling in the pathogenesis of CRVO. The observed beneficial effects of exogenous ADM suggest the endogenous up-regulation of $A d m$ is a compensatory response to CRVO. 

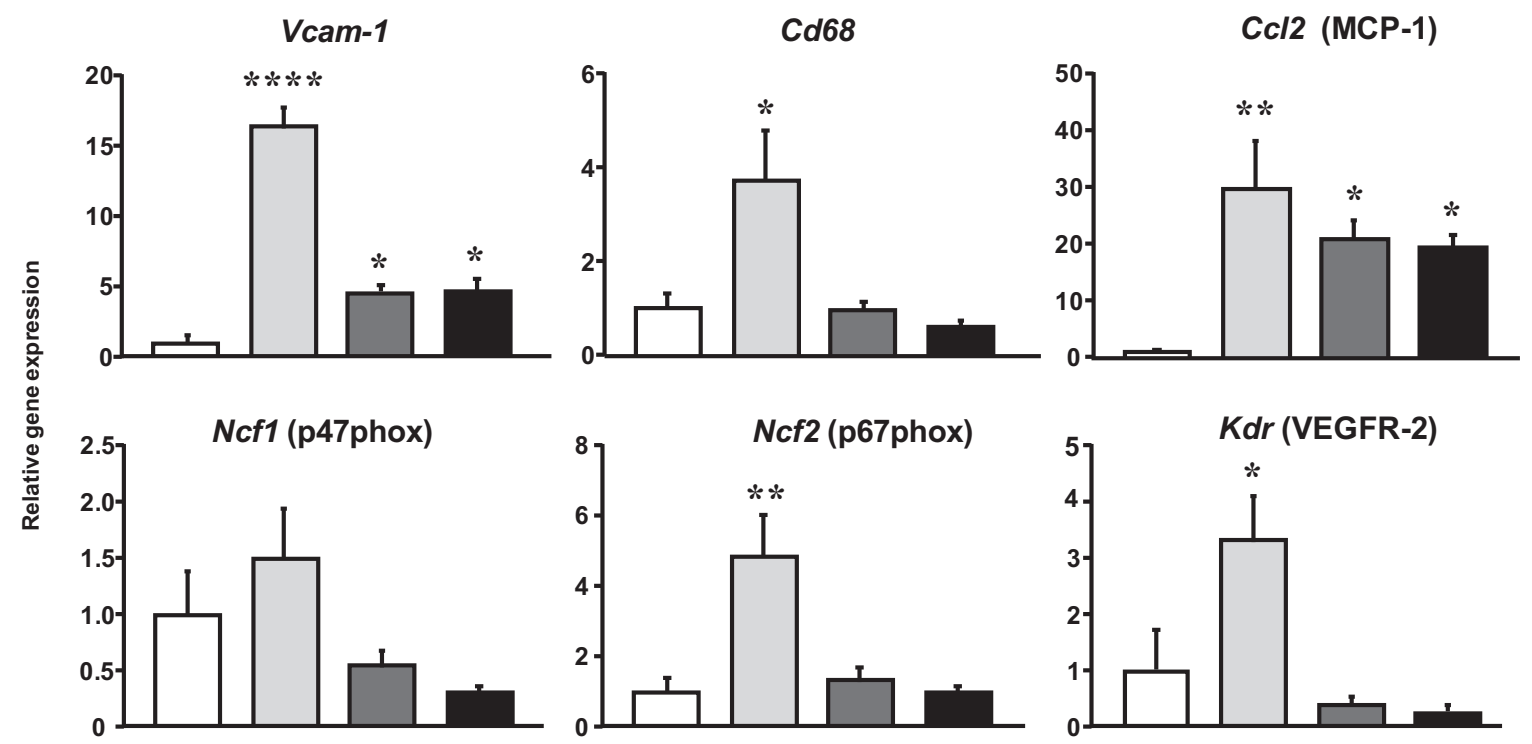

Figure 13 Adrenomedullin (ADM) directly suppresses retinal endothelial injury. Quantitative real-time PCR analysis showing relative expression of the indicated genes in TR-iBRB cells, an immortalized retinal capillary endothelial cell line. The mean of the control group (Ctrl) was assigned a value of 1. Endothelial injury was induced by exposing the cells to $20 \mathrm{ng} / \mathrm{mL}$ tumor necrosis factor (TNF)- $\alpha$ for 24 hours in the presence or absence of ADM (10 $0^{-7}$ or $10^{-9}$ mol/L). Data are expressed as means \pm SEM. $n=5$ to $7 .{ }^{*} P<0.05,{ }^{*} P<0.01$, and ${ }^{* * *} P<0.0001$ versus control (Dunnett's test). MCP, monocyte chemotactic protein; VEGFR, vascular endothelial growth factor receptor.

Damage to endothelial cells within retinal blood vessels may serve as the initial stimulus for platelet adhesion and coagulation, leading to vascular occlusion. ${ }^{27}$ Inflammation and oxidative stress are subsequently increased as the pathologic condition progresses toward later stages. ${ }^{46}$ Consistent with that scenario, the expression of the coagulation factor Serpinel (PAI-1) was elevated at an early stage after CRVO, whereas inflammatory and oxidative stress markers, such as Vcam1, Cd68, Ncf1 (p47phox), and Ncf2 (p67phox), were elevated at a later stage. Expression of these genes was higher in Adm KO than WT mice, suggesting a worsening of the pathologic condition in $A d m \mathrm{KO}$ mice. Conversely, ADM administration suppressed expression of these factors, which reflects amelioration of the pathologic condition. Similarly, the increase in CD68positive macrophages and activated microglia within retina was suppressed by ADM administration. Thus, ADM appears to suppress proinflammatory macrophage invasion and microglial activation after CVRO.

Interestingly, ADM administration also markedly suppressed the up-regulation of $K d r$ (VEGF receptor-2) after CRVO. Because VEGF signaling plays a critical role in the pathology of CRVO, which accounts for the efficacy of antiVEGF therapy currently used to treat CRVO patients, suppression of VEGF signaling by ADM is an encouraging result from a clinical viewpoint.

Using a retinal endothelial cell line, it was shown that ADM directly suppresses endothelial injury. Inflammationand oxidative stress-related genes and $K d r$ (VEGF receptor-2) expression were all increased by endothelial injury, and all were suppressed by ADM administration, which is consistent with the effects of ADM administration in the CRVO model.

Figure 14 summarizes the actions and therapeutic potential of the ADM-RAMP2 system in CRVO. Virchow's triad

\section{Virchow's triad}

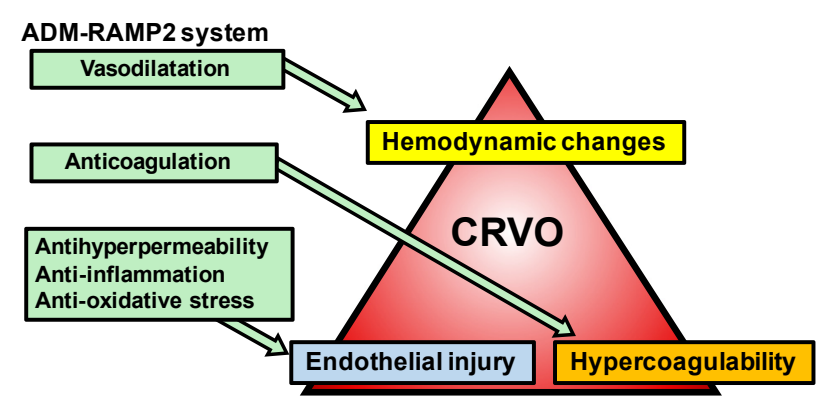

Figure 14 Summary of the actions and therapeutic potential of the adrenomedullin (ADM)-RAMP2 system in central retinal vein occlusion (CRV0). Virchow's triad describes the three elements (hemodynamic changes, endothelial injury, and hypercoagulability) that contribute to vascular thrombosis. ADM was originally identified as a vasodilating peptide and would be expected to improve reperfusion of vessels in which blood flow was interrupted by CRVO. ADM also exerts anticoagulation effects, which may suppress the hypercoagulability in CRV0. In addition, ADM suppresses inflammation and oxidative stress associated with CRVO, which coordinately promote endothelial injury. Finally, ADM suppresses vascular hyperpermeability, which is the cause of retinal edema. ADM appears to break Virchow's triad, making it a promising therapeutic target for the treatment of CRVO. 
includes the hemodynamic changes, endothelial injury, and hypercoagulability that contribute to vascular thrombosis. ADM was originally identified as a vasodilating peptide and would be expected to improve blood flow in CRVO. ADM also exerts anticoagulation effects, which may suppress the hypercoagulability in CRVO. Moreover, ADM suppresses inflammation and oxidative stress associated with CRVO, which would act coordinately to promote endothelial injury, and also suppresses vascular hyperpermeability, which is the cause of retinal edema. Thus, its ability to break Virchow's triad makes the ADM-RAMP2 system a promising therapeutic target for the treatment of CRVO.

Anti-VEGF antibodies are already being used as standard therapy for some retinal vascular diseases, and there is no doubt about their effectiveness. However, long-term administration of anti-VEGF antibodies sometimes leads to tachyphylaxis. ${ }^{4,5}$ Because the biological functions of ADM and anti-VEGF antibodies are different, coadministration of ADM and anti-VEGF antibodies may be an effective approach in some cases. ADM may also be useful as secondary therapy in patients who become resistant to anti-VEGF antibodies.

\section{Supplemental Data}

Supplemental material for this article can be found at https://doi.org/10.1016/j.ajpath.2018.10.021.

\section{References}

1. Rogers S, McIntosh RL, Cheung N, Lim L, Wang JJ, Mitchell P, Kowalski JW, Nguyen H, Wong TY: International Eye Disease Consortium: The prevalence of retinal vein occlusion: pooled data from population studies from the United States, Europe, Asia, and Australia. Ophthalmology 2010, 117:313-319.e1

2. O'Mahoney PR, Wong DT, Ray JG: Retinal vein occlusion and traditional risk factors for atherosclerosis. Arch Ophthalmol 2008, 126 : 692-699

3. Wong TY, Scott IU: Clinical practice: retinal-vein occlusion. N Engl J Med 2010, 363:2135-2144

4. Schaal S, Kaplan HJ, Tezel TH: Is there tachyphylaxis to intravitreal anti-vascular endothelial growth factor pharmacotherapy in age-related macular degeneration? Ophthalmology 2008, 115:2199-2205

5. Keane PA, Liakopoulos S, Ongchin SC, Heussen FM, Msutta S, Chang KT, Walsh AC, Sadda SR: Quantitative subanalysis of optical coherence tomography after treatment with ranibizumab for neovascular age-related macular degeneration. Invest Ophthalmol Vis Sci 2008, 49:3115-3120

6. Fujita Y, Mimata H, Nasu N, Nomura T, Nomura Y, Nakagawa M: Involvement of adrenomedullin induced by hypoxia in angiogenesis in human renal cell carcinoma. Int J Urol 2002, 9:285-295

7. Wang X, Yue TL, Barone FC, White RF, Clark RK, Willette RN, Sulpizio AC, Aiyar NV, Ruffolo RR Jr, Feuerstein GZ: Discovery of adrenomedullin in rat ischemic cortex and evidence for its role in exacerbating focal brain ischemic damage. Proc Natl Acad Sci U S A 1995, 92:11480-11484

8. Nguyen SV, Claycomb WC: Hypoxia regulates the expression of the adrenomedullin and HIF-1 genes in cultured HL-1 cardiomyocytes. Biochem Biophys Res Commun 1999, 265:382-386
9. Shindo T, Kurihara Y, Nishimatsu H, Moriyama N, Kakoki M, Wang Y, Imai Y, Ebihara A, Kuwaki T, Ju KH, Minamino N, Kangawa K, Ishikawa T, Fukuda M, Akimoto Y, Kawakami H, Imai T, Morita H, Yazaki Y, Nagai R, Hirata Y, Kurihara H: Vascular abnormalities and elevated blood pressure in mice lacking adrenomedullin gene. Circulation 2001, 104:1964-1971

10. Iimuro S, Shindo T, Moriyama N, Amaki T, Niu P, Takeda N, Iwata H, Zhang Y, Ebihara A, Nagai R: Angiogenic effects of adrenomedullin in ischemia and tumor growth. Circ Res 2004, 95:415-423

11. Brain SD, Grant AD: Vascular actions of calcitonin gene-related peptide and adrenomedullin. Physiol Rev 2004, 84:903-934

12. Kato J, Tsuruda T, Kita T, Kitamura K, Eto T: Adrenomedullin: a protective factor for blood vessels. Arterioscler Thromb Vasc Biol 2005, 25:2480-2487

13. Shimosawa T, Ogihara T, Matsui H, Asano T, Ando K, Fujita T: Deficiency of adrenomedullin induces insulin resistance by increasing oxidative stress. Hypertension 2003, 41:1080-1085

14. McLatchie LM, Fraser NJ, Main MJ, Wise A, Brown J, Thompson N, Solari R, Lee MG, Foord SM: RAMPs regulate the transport and ligand specificity of the calcitonin-receptor-like receptor. Nature 1998, 393:333-339

15. Parameswaran N, Spielman WS: RAMPs: the past, present and future. Trends Biochem Sci 2006, 31:631-638

16. Ichikawa-Shindo Y, Sakurai T, Kamiyoshi A, Kawate H, Iinuma N, Yoshizawa T, Koyama T, Fukuchi J, Iimuro S, Moriyama N, Kawakami H, Murata T, Kangawa K, Nagai R, Shindo T: The GPCR modulator protein RAMP2 is essential for angiogenesis and vascular integrity. J Clin Invest 2008, 118:29-39

17. Okamura T, Ayajiki K, Kangawa K, Toda N: Mechanism of adrenomedullin-induced relaxation in isolated canine retinal arteries. Invest Ophthalmol Vis Sci 1997, 38:56-61

18. Dorner GT, Garhofer G, Huemer KH, Golestani E, Zawinka C, Schmetterer L, Wolzt M: Effects of adrenomedullin on ocular hemodynamic parameters in the choroid and the ophthalmic artery. Invest Ophthalmol Vis Sci 2003, 44:3947-3951

19. Er H, Doganay S, Ozerol E, Yurekli M: Adrenomedullin and leptin levels in diabetic retinopathy and retinal diseases. Ophthalmologica 2005, 219:107-111

20. Ito S, Fujisawa K, Sakamoto T, Ishibashi T: Elevated adrenomedullin in the vitreous of patients with diabetic retinopathy. Ophthalmologica 2003, 217:53-57

21. Lu Y, Xu Y, Tang C: Changes in adrenomedullin in patients with proliferative diabetic retinopathy. Curr Eye Res 2011, 36:1047-1052

22. Nakamura T, Honda K, Ishikawa S, Kitamura K, Eto T, Saito T: Plasma adrenomedullin levels in patients with non-insulin dependent diabetes mellitus: close relationships with diabetic complications. Endocr J 1998, 45:241-246

23. Iesato $Y$, Toriyama $Y$, Sakurai T, Kamiyoshi A, Ichikawa-Shindo $Y$, Kawate H, Yoshizawa T, Koyama T, Uetake R, Yang L, Yamauchi A, Tanaka M, Igarashi K, Murata T, Shindo T: Adrenomedullin-RAMP2 system is crucially involved in retinal angiogenesis. Am J Pathol 2013, 182:2380-2390

24. Iesato Y, Yuda K, Chong KT, Tan X, Murata T, Shindo T, Yanagi Y: Adrenomedullin: a potential therapeutic target for retinochoroidal disease. Prog Retin Eye Res 2016, 52:112-129

25. Imai A, Toriyama $\mathrm{Y}$, Iesato $\mathrm{Y}$, Hirabayashi $\mathrm{K}$, Sakurai $\mathrm{T}$, Kamiyoshi A, Ichikawa-Shindo Y, Kawate H, Tanaka M, Liu T, Xian X, Zhai L, Dai K, Tanimura K, Liu T, Cui N, Yamauchi A, Murata T, Shindo T: Adrenomedullin suppresses vascular endothelial growth factor-induced vascular hyperpermeability and inflammation in retinopathy. Am J Pathol 2017, 187:999-1015

26. Khayat M, Lois N, Williams M, Stitt AW: Animal models of retinal vein occlusion. Invest Ophthalmol Vis Sci 2017, 58:6175-6192

27. Zhang H, Sonoda KH, Qiao H, Oshima T, Hisatomi T, Ishibashi T: Development of a new mouse model of branch retinal vein occlusion and retinal neovascularization. Jpn J Ophthalmol 2007, 51: $251-257$ 
28. Paques M, Guyomard JL, Simonutti M, Roux MJ, Picaud S, Legargasson JF: Sahel JA: Panretinal, high-resolution color photography of the mouse fundus. Invest Ophthalmol Vis Sci 2007, 48: 2769-2774

29. Roszer T: Understanding the mysterious M2 macrophage through activation markers and effector mechanisms. Mediators Inflamm 2015 2015:816460

30. Hayreh SS: Management of central retinal vein occlusion. Ophthalmologica 2003, 217:167-188

31. McIntosh RL, Rogers SL, Lim L, Cheung N, Wang JJ, Mitchell P, Kowalski JW, Nguyen HP, Wong TY: Natural history of central retinal vein occlusion: an evidence-based systematic review. Ophthalmology 2010, 117:1113-1123.e15

32. Sawada O, Ohji M: Retinal vein occlusion. Dev Ophthalmol 2016, 55 : $147-153$

33. Inoue M, Kobayakawa S, Sotozono C, Komori H, Tanaka K, Suda Y, Matsushima H, Kinoshita S, Senoo T, Tochikubo T, Kadonosono K: Evaluation of the incidence of endophthalmitis after intravitreal injection of anti-vascular endothelial growth factor. Ophthalmologica 2011, 226:145-150

34. Grunwald JE, Pistilli M, Ying GS, Maguire MG, Daniel E, Martin DF Growth of geographic atrophy in the comparison of age-related macular degeneration treatments trials. Ophthalmology 2015, 122: 809-816

35. Kolar P: Risk factors for central and branch retinal vein occlusion: a meta-analysis of published clinical data. J Ophthalmol 2014, 2014: 724780

36. Ebneter A, Agca C, Dysli C, Zinkernagel MS: Investigation of retinal morphology alterations using spectral domain optical coherence tomography in a mouse model of retinal branch and central retinal vein occlusion. PLoS One 2015, 10:e0119046

37. Banks JG, Board RG, Carter J, Dodge AD: The cytotoxic and photodynamic inactivation of micro-organisms by Rose Bengal. J Appl Bacteriol 1985, 58:391-400

38. Goldenberg-Cohen $\mathrm{N}$, Dadon S, Avraham BC, Kramer M, Hasanreisoglu M, Eldar I, Weinberger D, Bahar I: Molecular and histological changes following central retinal artery occlusion in a mouse model. Exp Eye Res 2008, 87:327-333

39. Ben-Nun J: Capillary blood flow in acute branch retinal vein occlusion. Retina 2001, 21:509-512

40. MacDonald D: The ABCs of RVO: a review of retinal venous occlusion. Clin Exp Optom 2014, 97:311-323

41. Hayreh SS, van Heuven WA, Hayreh MS: Experimental retinal vascular occlusion, I: pathogenesis of central retinal vein occlusion. Arch Ophthalmol 1978, 96:311-323

42. Wilson CA, Hatchell DL: Photodynamic retinal vascular thrombosis: rate and duration of vascular occlusion. Invest Ophthalmol Vis Sci 1991, 32:2357-2365

43. Schmidt D: Comorbidities in combined retinal artery and vein occlusions. Eur J Med Res 2013, 18:27

44. Miyamoto K, Ogura Y: Pathogenetic potential of leukocytes in diabetic retinopathy. Semin Ophthalmol 1999, 14:233-239

45. Thach AB, Yau L, Hoang C, Tuomi L: Time to clinically significant visual acuity gains after ranibizumab treatment for retinal vein occlusion: BRAVO and CRUISE trials. Ophthalmology 2014, 121:1059-1066

46. Noma H, Mimura T, Eguchi S: Association of inflammatory factors with macular edema in branch retinal vein occlusion. JAMA Ophthalmol 2013, 131:160-165 